\title{
Broadband enhancement of light-matter interaction in photonic crystal cavities integrating site-controlled quantum dots
}

\author{
Marco Felici $\odot,{ }^{1, *}$ Giorgio Pettinari, ${ }^{2}$ Francesco Biccari, ${ }^{3}$ Alice Boschetti, ${ }^{3}$ Saeed Younis, ${ }^{1, \dagger}$ Simone Birindelli, ${ }^{1}$ \\ Massimo Gurioli, ${ }^{3}$ Anna Vinattieri, ${ }^{3}$ Annamaria Gerardino, ${ }^{2}$ Luca Businaro, ${ }^{2}$ Mark Hopkinson, ${ }^{4}$ \\ Silvia Rubini, ${ }^{5}$ Mario Capizzi, ${ }^{1}$ and Antonio Polimeni ${ }^{1}$ \\ ${ }^{1}$ Department of Physics, Sapienza University of Rome, Piazzale Aldo Moro 5, I-00185 Roma, Italy \\ ${ }^{2}$ National Research Council (CNR), Institute for Photonics and Nanotechnologies, (IFN-CNR), Via Cineto Romano 42, I-00156 Roma, Italy \\ ${ }^{3}$ Department of Physics and Astronomy, University of Florence, Via Giovanni Sansone 1, I-50019 Sesto Fiorentino (FI), Italy \\ and European Laboratory for Non-Linear Spectroscopy (LENS), University of Florence, Via Nello Carrara 1, \\ I-50019 Sesto Fiorentino (FI), Italy \\ ${ }^{4}$ Department of Electronic and Electrical Engineering, University of Sheffield, Sheffield S3 3JD, United Kingdom \\ ${ }^{5}$ IOM-CNR, Laboratorio TASC, Area Science Park, Strada Statale 14, Km 163.5, 34149 Trieste, Italy
}

(Received 26 July 2019; revised manuscript received 28 March 2020; accepted 1 April 2020; published 5 May 2020)

\begin{abstract}
The fabrication of integrated quantum dot (QD)-optical microcavity systems is a requisite step for the realization of a wide range of nanophotonic experiments (and applications) that exploit the ability of QDs to emit nonclassical light, e.g., single photons. Thanks to their $\sim 20$-nm positioning accuracy and to their proven potential for single-photon operation, the QDs obtained by spatially selective hydrogen irradiation of dilute-nitride semiconductors — such as $\mathrm{Ga}(\mathrm{AsN})$ and $\mathrm{Ga}(\mathrm{PN})$ - are uniquely suited for integration with photonic nanodevices. In the present work, we demonstrate the ability to deterministically integrate single, site-controlled $\mathrm{Ga}(\mathrm{AsN}) / \mathrm{Ga}(\mathrm{AsN}): \mathrm{H}$ QDs within a photonic crystal (PhC) cavity. The properties of the fabricated QD-PhC cavity systems are then probed by photon correlation — providing clear evidence of single-photon emission-and time-resolved microphotoluminescence spectroscopy. Detailed information on the dynamics of our integrated nanodevices can be inferred by comparing these experiments to the solutions of a rate-equations system, developed by taking into account all the main processes leading to the capture, relaxation, and recombination of carriers in and out of the QD. This allows us to follow the evolution of the relevant recombination rates in our system for varying energy detuning, $\Delta E$, between the QD and the PhC cavity. When the QD exciton transition is nearly resonant with the cavity mode, a large (>tenfold) enhancement of the spontaneous emission rate is observed, in substantial agreement with Jaynes-Cummings (JC) theory. For intermediate detunings ( $\Delta E \sim 1.5-3.5 \mathrm{meV}$ ), on the other hand, the observed enhancement is significantly larger than that predicted by JC theory, due to the important role played by acoustic phonons in mediating the QD-PhC cavity coupling in a solid-state environment. Apart from its fundamental interest, the observation of such phonon-mediated, broadband enhancement of light-matter interaction significantly relaxes the requirements for the realization of a large variety of cavity QED-based experiments and applications. These include many photonic devices for which the use of site-controlled $\mathrm{Ga}(\mathrm{AsN}) / \mathrm{Ga}(\mathrm{AsN}): \mathrm{H}$ QDs would be inherently advantageous, such as those based on the coupling between more than one QD and a single cavity mode (e.g., few-QD nanolasers and QD solids).
\end{abstract}

DOI: 10.1103/PhysRevB.101.205403

\section{INTRODUCTION}

In recent years, semiconductor quantum dots (QDs) have started to attract considerable interest for the realization of triggered sources of nonclassical light, wherein exactly one photon [1-3] — or one entangled photon pair [4-8] - is produced for each excitation pulse. The potential for triggered operation, however, only makes up part of the appeal of QD-based light emitters; another important aspect is represented by the possibility of monolithically integrating them with complex nanophotonic circuits [9-11], embedding all

\footnotetext{
*marco.felici@ roma1.infn.it
}

${ }^{\dagger}$ Deceased. the elements required for the emission, manipulation, and detection of photons on the same semiconductor chip. Such fully integrated devices could serve as the building blocks of future optical quantum computing schemes [12], and their realization would represent an important milestone in the development of quantum information technology.

Nevertheless, the deterministic integration of one or more QDs in a nanophotonic device is a challenging task, which requires full control over the QD position and emission energy. Even the coupling of a single QD with, e.g., an optical microcavity [13-18] demands a very precise spatial—within $50 \mathrm{~nm}$ - and spectral - better than 1-2 meV-matching of the embedded QDs with the confined microcavity modes, based on the expectations of Jaynes-Cummings (JC) theory [19]. In order to meet these requirements, several methods for 
defining the QD position and template by lithographic means have been proposed and developed in the last decade [20-29]. Recently, a novel route to the fabrication of site-controlled nanostructures - based on $\mathrm{H}$-assisted defect engineering in dilute nitride semiconductors, such as $\mathrm{Ga}(\mathrm{AsN})$ and $\mathrm{Ga}(\mathrm{PN})$ has been demonstrated [30-33]. Owing to the formation of stable $\mathrm{N}-n \mathrm{H}$ complexes $(n \geqslant 2)$ [34,35], indeed, H irradiation of these materials neutralizes all the effects of $\mathrm{N}$ incorporation, including the large, $\mathrm{N}$-induced band-gap reduction [36-41]. Therefore, the deposition of lithographically defined, $\mathrm{H}$-opaque masks prior to hydrogenation leads to the formation of nanometer-sized potential wells, i.e., QDs, which have recently shown evidence of triggered single-photon emission [42]. It is also worth mentioning here that spatially selective $\mathrm{H}$ removal-e.g., by laser annealing performed with a nearfield optical microscope [43] or with the aid of plasmonic bow-tie nanoapertures [44] - has also been demonstrated as a viable alternative for the realization of energy tunable, sitecontrolled $\mathrm{Ga}(\mathrm{AsN}) / \mathrm{Ga}(\mathrm{AsN}): \mathrm{H}$ QDs, which have recently shown the ability to emit single photons [43].

In the present work, we successfully demonstrate the deterministic integration of the $\mathrm{Ga}(\mathrm{AsN}) / \mathrm{Ga}(\mathrm{AsN}): \mathrm{H}$ QDs fabricated by spatially selective $\mathrm{H}$ irradiation with photonic crystal (PhC) microcavities, and we analyze the effects of QDcavity coupling on the recombination dynamics of the system. As detailed in the upcoming discussion, we indeed observe a $\sim$ tenfold variation in the recombination rate of the QD exciton $(\mathrm{X})$ as a function of the energy detuning, $\Delta E$, between the $\mathrm{X}$ transition and the fundamental cavity mode (CM). Moreover, the experimentally determined functional dependence of the QD recombination rate on $\Delta E$ significantly deviates from the expectations of the JC model [19], hinting at the importance of phonon dephasing for the determination of the emittercavity coupling strength in solid-state systems $[45,46]$. This topic has been attracting a lot of attention recently, as it is related to the observation of non-Markovian dynamics [47-49] and of anomalously large Purcell-enhancement rates in mildly detuned ( $\Delta E \leqslant 5 \mathrm{meV}$ ) QD-PhC cavity systems [16-18]. Apart from their fundamental interest, both of these phenomena are relevant for the design of quantum information protocols based on QD-PhC cavity systems. Also, the observation of such delicate cavity quantum electrodynamics (QED) effects further validates the intrinsic suitability of $\mathrm{Ga}(\mathrm{AsN}) / \mathrm{Ga}(\mathrm{AsN}): \mathrm{H}$ QDs for the integration with photonic devices, and it represents an excellent starting point for the realization of more complex photonic circuits based on these site-controlled nanostructures.

\section{RESULTS AND DISCUSSION}

\section{A. Fabrication and emission properties of integrated QD-PhC cavity systems}

As sketched in Fig. 1, the steps required for the fabrication of an integrated $\mathrm{Ga}(\mathrm{AsN}) / \mathrm{Ga}(\mathrm{AsN}): \mathrm{H}$ QD-PhC cavity system start off by using electron-beam lithography (EBL) to pattern the surface of a $\mathrm{Ga}(\mathrm{AsN})$ sample with H-opaque, hydrogen silsesquioxane (HSQ) masks. The sample in question is a $\mathrm{Ga}(\mathrm{AsN})$ quantum well (QW) grown by molecular-beam epitaxy (MBE), having a $\mathrm{N}$ concentration $x=1.1 \%$ and a

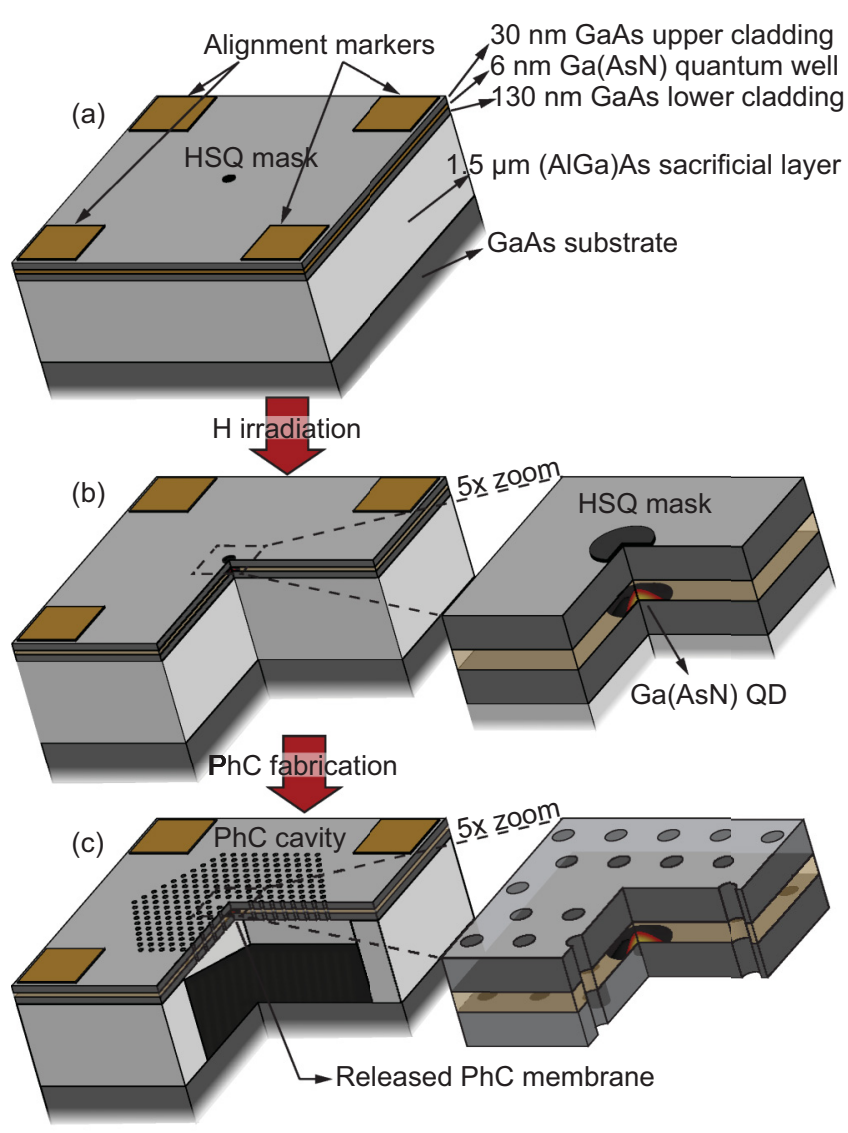

FIG. 1. Sketch of the processing steps required for the deterministic integration of a single $\mathrm{Ga}(\mathrm{AsN}) / \mathrm{Ga}(\mathrm{AsN}): \mathrm{H}$ QD with a $\mathrm{PhC}$ cavity (the elements displayed in the sketch are not in scale). (a) A pattern of HSQ masks, defined by EBL, is deposited on the sample surface (the layer sequence of the sample is indicated in the figure; see also the main text). The HSQ masks are aligned to a set of metallic ( $\mathrm{Cr}-\mathrm{Au})$ markers, previously defined by EBL. (b) Following $\mathrm{H}$ irradiation (and, subsequently, $\mathrm{H}$ diffusion inside the sample) a site-controlled QD forms underneath each H-opaque mask. (c) A PhC cavity is fabricated around each $\mathrm{Ga}(\mathrm{AsN}) / \mathrm{Ga}(\mathrm{AsN}): \mathrm{H}$ QD. The reference system defined by the metallic markers ensures a near-perfect ( $\sim 20 \mathrm{~nm}$ accuracy) alignment between the QD and the center of the PhC cavity.

thickness $t_{\mathrm{QW}}=6 \mathrm{~nm}$. The $\mathrm{QW}$ is sandwiched between a 130-nm-thick lower cladding and a 30-nm-thick capping layer, both made of GaAs. These three layers are grown on top of a 1.5- $\mu \mathrm{m}$-thick sacrificial (AlGa)As layer (Al concentration equal to $70 \%$ ), which can be removed by diluted-HF (5\%) wet etching to create a suspended dielectric membrane integrating the fabricated photonic structure (see also Ref. [50]). It is important to note that [as shown in Fig. 1(a)] the deposited HSQ masks are aligned with a series of chromium-gold $(\mathrm{Cr}-$ $\mathrm{Au}$ ) reference markers-fabricated by EBL and lift-off- to enable the deterministic positioning of each QD within the final photonic structure. The desired pattern of site-controlled $\mathrm{Ga}(\mathrm{AsN}) / \mathrm{Ga}(\mathrm{AsN}): \mathrm{H}$ QDs is then obtained by irradiating the masked sample surface with low-energy $(100 \mathrm{eV}) \mathrm{H}^{+}$ions (hydrogen dose $=1 \times 10^{17}$ ions $/ \mathrm{cm}^{2}$, hydrogenation temperature $=190^{\circ} \mathrm{C}$ ), which diffuse inside $\mathrm{Ga}(\mathrm{AsN})$ with a typical steplike profile (the concentration of $\mathrm{H}$ decreases by one order 
of magnitude within a depth of $5 \mathrm{~nm}$ ) [51]. The sharpness of this diffusion front-coupled to the fact that the capping layer is kept thin enough $(30 \mathrm{~nm})$ to minimize any "smearing" of the $\mathrm{H}$ profile due to Fick-like diffusion in $\mathrm{N}$-free GaAs [51] - implies that the template defined by the masks at the sample surface is closely reproduced by the diffusing $\mathrm{H}^{+}$ ions; for circular masks, such as those used in this work, this results in the formation of a single, site-controlled QD underneath each mask [see Fig. 1(b)], provided that the correct mask size, $d_{\mathrm{M}}$, is employed (the dependence of the properties of the fabricated $\mathrm{Ga}(\mathrm{AsN}) / \mathrm{Ga}(\mathrm{AsN}): \mathrm{H}$ nanostructures on $d_{\mathrm{M}}$ was illustrated in Ref. [31]). Since such optimal size depends on the specific sample and on the impinging $\mathrm{H}$ dose, $d_{\mathrm{H}}$, each lithographically defined pattern is designed to contain a fairly large number of different dot mask sizes, i.e., $d_{\mathrm{M}}=90$, $100,120,160,200,350$, and $500 \mathrm{~nm}$. This ensures that, for a given $d_{\mathrm{H}}$, at least one mask size will yield suitable QDs (e.g., for the sample discussed in the present work, irradiation with $d_{\mathrm{H}}=1 \times 10^{17}$ ions $/ \mathrm{cm}^{2}$ typically resulted in the formation of QDs underneath masks with $d_{\mathrm{M}}=120$ and $160 \mathrm{~nm}$ ). Thanks to the aforementioned $\mathrm{Cr}$-Au reference markers, it is then possible to fabricate $L_{N} \mathrm{PhC}$ cavities [52] [with $N=3,7$, or 11 holes missing from the PhC lattice; the sketch in Fig. 1(c) refers to an $L_{3}$ cavity] precisely positioned around each dot (realignment precision of the EBL system $\sim 20 \mathrm{~nm}$ ). The desired hole pattern is first defined by EBL on a layer of ZEP520A resist, and then transferred into the $\mathrm{GaAs} / \mathrm{Ga}(\mathrm{AsN}) / \mathrm{GaAs}$ membrane by chlorine-based dry etching. Finally, as mentioned above, the membrane is released by etching away the sacrificial (AlGa)As layer. For fixed values of the $\mathrm{PhC}$ lattice constant $a$ and hole radius $r$ (with the latter usually expressed in terms of the dimensionless $r / a$ ratio), the cavity-mode frequencies of $L_{N}$ cavities obviously depend on the cavity size, i.e., on $N$ [53,54]. Therefore, $a$ and/or the $r / a$ ratio must be carefully adjusted in order to spectrally match each cavity type with the average QD energy. In order to aid such "lithographic tuning" of the CM frequencies, we performed guided-mode expansion calculations [55] of the $\mathrm{PhC}$ structures to be included in the fabricated sample. However, the unavoidable approximations that must be made in such calculations (for example, the assumption of a real, frequency-independent dielectric constant for the material) virtually ensure the presence of small discrepancies between the computed and experimentally measured CM frequencies. Also, while the spatial resolution of our electron-beam lithography system $(\sim 4 \mathrm{~nm}[50])$ allows one to obtain values of the hole radii that are very uniform across the sample, the uncertainty inherent to the etching steps of the fabrication process often results in a small systematic error in the absolute value of the r/a ratio, and thus of the CM frequencies. In order to account for these uncertainties, and also for the spread in the emission energy of the fabricated QDs-typically of the order of the full width at half maximum (FWHM) of the original $\mathrm{Ga}(\mathrm{AsN}) / \mathrm{GaAs} \mathrm{QW}, \sim 20 \mathrm{meV}$ [43] - the fabrication of all $\mathrm{PhC}$ structures was repeated for values of $a$ ranging between 215 and $255 \mathrm{~nm}$ (in steps of $10 \mathrm{~nm}$ ), whereas the $r / a$ ratio was kept fixed to 0.29 for all the structures. This procedure enabled us to have at least a few spectrally matched devices for each type of fabricated $\mathrm{PhC}$ cavity, without any prior calibration of the CM energy with respect to the QD
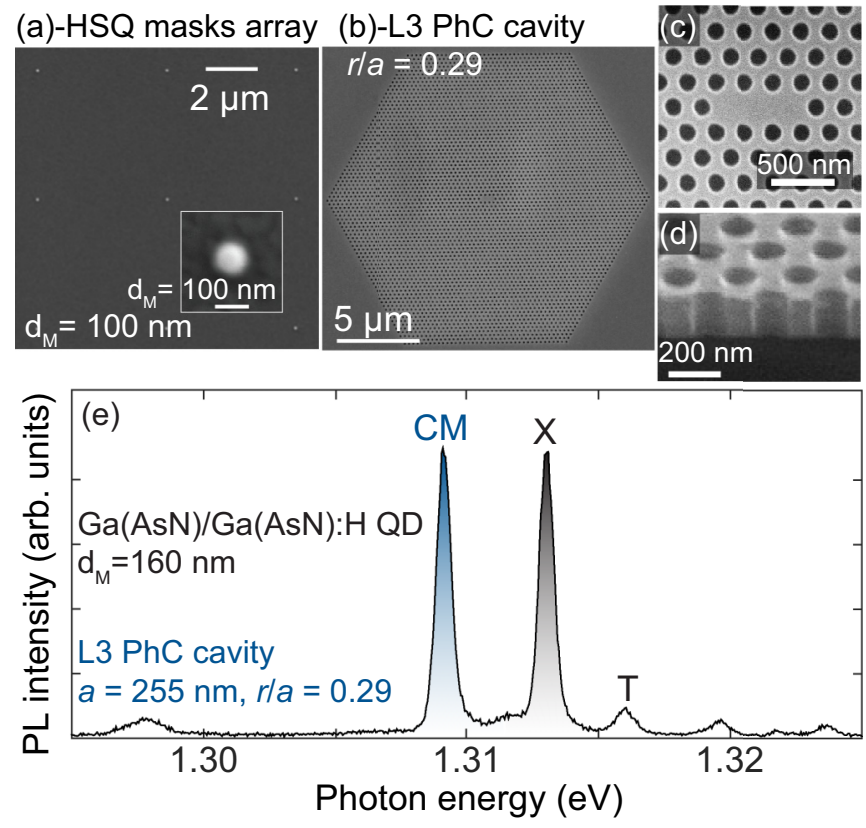

FIG. 2. (a) SEM image of an array of circular HSQ masks $\left(d_{\mathrm{M}}=\right.$ $100 \mathrm{~nm}$, dot-dot spacing equal to $5 \mu \mathrm{m}$ ) ready for the fabrication of $\mathrm{Ga}(\mathrm{AsN}) / \mathrm{Ga}(\mathrm{AsN}): \mathrm{H}$ QDs. Inset: Magnified view of a single HSQ mask $\left(d_{\mathrm{M}}=100 \mathrm{~nm}\right)$. (b) SEM image of a typical fabricated $L_{3} \mathrm{PhC}$ cavity, with lattice parameter $a=255 \mathrm{~nm}$ and $r / a$ ratio equal to 0.29 . (c) Zoom-in of the cavity region for a device similar to the one shown in (b). (d) Cross-sectional view of a fabricated PhC cavity, showing the released GaAs membrane embedding a single site-controlled $\mathrm{Ga}(\mathrm{AsN}) / \mathrm{Ga}(\mathrm{AsN}): \mathrm{H}$ QD (not visible in the image). (e) Micro-PL spectrum of a QD-PhC device (temperature $T=10 \mathrm{~K}$, excitation power density $P_{e x}=0.3 \mathrm{~kW} / \mathrm{cm}^{2}$ ), showing the line corresponding to the $\mathrm{X}$ transition (the area underneath the peak was filled in with black for a better visualization) and the resonance associated with the fundamental CM (blue-filled area). The peak labeled as " $\mathrm{T}$ " is attributed to a charged exciton (trion).

emission. It goes without saying that in samples devised with a specific application in mind-rather than for "proof-ofprinciple" studies, such as this one-the number of spectrally coupled devices could be significantly increased by taking a few straightforward measures, e.g., by settling on a specific $\mathrm{PhC}$ cavity type and/or by narrowing down the range of $\mathrm{PhC}$ lattice constants included in the lithographic patterns.

Figures 2(a) and 2(b)-2(d) show, respectively, scanningelectron microscope (SEM) images of an array of circular HSQ masks-ready for the fabrication of ordered $\mathrm{Ga}(\mathrm{AsN}) / \mathrm{Ga}(\mathrm{AsN}): \mathrm{H}$ QDs-and of a fabricated $\mathrm{PhC} L_{3}$ cavity $(a=255 \mathrm{~nm}, r / a=0.29)$. For the array displayed in Fig. 2(a) the mask spacing was set to $5 \mu \mathrm{m}$ (mask diameter $d_{\mathrm{M}}=100 \mathrm{~nm}$ ), an interdot distance ideal for the characterization of single QDs by microphotoluminescence (micro-PL). For the realization of integrated QD-PhC cavity systems, on the other hand, we opted for a much larger mask spacing (35 $\mu \mathrm{m})$, to ensure that each cavity [contained within a hexagonal $\mathrm{PhC}$ lattice having maximal diameter $=20 \mu \mathrm{m}$; see Fig. 2(b)] encapsulated a single QD. It is worth noting here that such freedom in QD positioning is a unique feature of our nanofabrication technique, which sets it apart from 
most other methods for the realization of site-controlled QDs. In QD growth, indeed, the density of formation/nucleation sites is deeply intertwined with other QD properties, such as the dot size and emission energy [56,57]. This typically remains true whenever the arrangement/density of the QD formation sites is defined by substrate patterning prior to epitaxial growth, as in the cases presented in Refs. [20-29]. As a consequence, it is usually quite difficult to place a QD in its prescribed position, e.g., in a photonic device, without having its emission energy affected by its distance from the other dots $[20,58] . \mathrm{Ga}(\mathrm{AsN}) / \mathrm{Ga}(\mathrm{AsN}): \mathrm{H}$ QDs, on the other hand, are fabricated postgrowth, so that their emission energy — which can be tuned by adjusting the mask size and the $\mathrm{H}$ dose, as noted above [31,42] —is in principle fully independent of their position. With respect to other methods for the fabrication of site-controlled nanostructures, another potential advantage of spatially selective $\mathrm{H}$ irradiation lies in the fact that this technique does not affect the sample morphology in any way. This implies that, after removing the H-opaque mask used to define the QD position - which is usually done before the $\mathrm{PhC}$ cavity is fabricated - the sample surface remains perfectly flat. This contrasts with other nanofabrication methods, which often feature either "bumps" [23] or "dips" [14] in coincidence with the dot position, potentially affecting the performance of the optical microcavities in which the QDs are integrated.

The micro-PL spectrum of a typical QD- $L_{3}$ PhC cavity system (dot mask diameter $d_{\mathrm{M}}=160 \mathrm{~nm}, \mathrm{PhC}$ lattice constant $a=255 \mathrm{~nm}$, and hole radius $r=74 \mathrm{~nm}$, corresponding to an $r / a$ ratio equal to 0.29) is displayed in Fig. 2(e). Micro-PL measurements were performed by placing the sample in a lowvibration He-flow cryostat, mounted on a two-axis positioning system based on high-precision (typical step size $=250 \mathrm{~nm}$ ) stepping motors. The PL signal was excited with a pulsed ( $\sim 200$ fs pulse width, $12.5 \mathrm{~ns}$ repetition period) Ti:sapphire laser tuned at a wavelength of $780 \mathrm{~nm}$ and frequency doubled with a BBO nonlinear crystal to reach an excitation wavelength $\lambda_{\mathrm{ex}}=390 \mathrm{~nm}$. The excitation laser was focused on the sample with a $100 \times$ microscope objective (numerical aperture $=0.7$, spot size $\sim 350 \mathrm{~nm}$ ); the resulting PL signal was collected through the same objective, analyzed with a $30-\mathrm{cm}$ monochromator mounting a $600 \mathrm{~g} / \mathrm{mm}$ grating, and detected with a Si charge-coupled device (CCD) detector (Princeton Instruments PIXIS 100F). The spectral resolution of this setup, directly estimated by averaging the values of the full width at half maximum (FWHM) measured for several lines of a calibration Hg lamp, is equal to $300 \mu \mathrm{eV}$.

The micro-PL spectrum reported in Fig. 2(e) is dominated by the features associated with the single-exciton recombination and with the fundamental mode of the $\mathrm{PhC}$ cavity. The latter is characterized by a quality factor $Q=2500$, consistent with the theoretical expectations for a standard $L_{3}$ cavity [14]. A third, weaker feature, labeled as " $T$ " and tentatively assigned to a charged exciton (trion), is also visible in the spectrum. At the temperature of the measurements presented in Fig. 2(e), $T=10 \mathrm{~K}$, the $\mathrm{X}$ transition is blueshifted by $\Delta E=3.9 \mathrm{meV}$ with respect to the cavity mode, whereas the $\mathrm{T}$ peak lies even further away, at $\sim 7 \mathrm{meV}$ from the $\mathrm{CM}$.

As summarized in Fig. 3, the excitonic transitions of the QD can be progressively tuned into resonance with the CM by sweeping the temperature of the sample [14,59]. This is

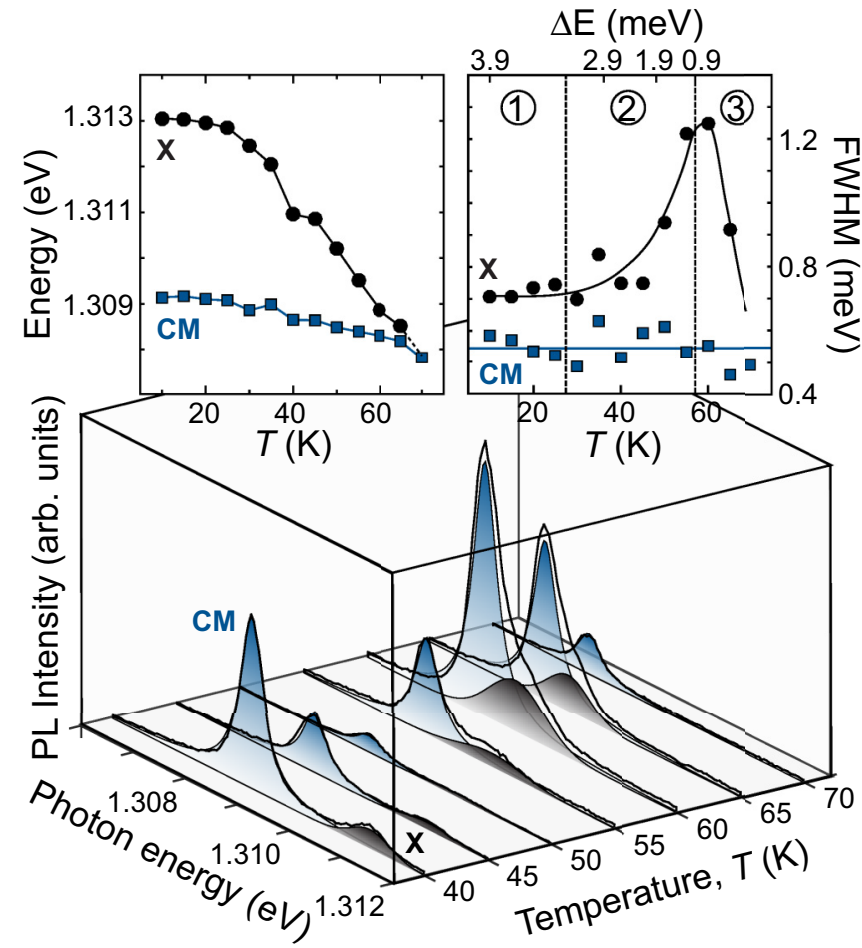

FIG. 3. (Main) Evolution of the micro-PL spectrum of the investigated QD-PhC cavity system $\left(P_{e x}=0.3 \mathrm{~kW} / \mathrm{cm}^{2}\right)$ as the temperature, $T$, is increased between 40 and $70 \mathrm{~K}$. The color-filled area plots displayed in the figure are fits to the experimental peaks (Gaussianshaded in black - for the $\mathrm{X}$ transition, Lorentzian - shaded in bluefor the CM line). Insets: $T$ dependence (in the $10-70 \mathrm{~K}$ range) of the peak position (left) and of the full width at half maximum (FWHM, right) of the $\mathrm{X}$ transition (black dots) and of the CM line (blue squares), as extracted from the fits reported in the main panel. The plot reported in the right inset is divided into three temperature intervals - labeled as 1-3-based on the criteria defined in the main text.

made possible by the much stronger $T$ dependence of the energy of the QD states-which follows the trend dictated by the $\mathrm{Ga}(\mathrm{AsN})$ band-gap shrinkage [60] — with respect to that of the CM (see left inset of Fig. 3), which decreases at an approximately linear rate $(\sim 20 \mu \mathrm{eV} / \mathrm{K}$, also consistent with Refs. [14,59]) due to the variation of the refractive index of GaAs with $T$. For the QD-PhC cavity system investigated here, however, it is important to note that the $\mathrm{X}$ line is the only QD transition showing a significant coupling with the CM. The only other realistic candidate, the T line [see Fig. 2(e)], likely remains too far off the $\mathrm{CM}$ within the investigated temperature range. Moreover, as $T$ increases-i.e., as the $\mathrm{T}-\mathrm{CM}$ detuning is reduced - the $\mathrm{T}$ line quickly disappears from the spectrum: it is, indeed, notably absent from the PL spectra displayed in the main panel of Fig. 3 (which cover the 40-70 K range), possibly due to temperature-induced changes in the charge environment surrounding the QD. As a result, in our data we do not see any evidence of the nonmonotonic temperature dependence of the CM energy (see left inset of Fig. 3) observed in Ref. [61] and attributed to the dragging/pulling of the $\mathrm{CM}$ as it successively enters into resonance with different excitonic species. As we will discuss in the 
next section, this experimental fact has important implications for the description of the QD dynamics, as it allows devising a model wherein a single state of the QD-the exciton-is coupled to the cavity mode.

The main panel of Fig. 3 also displays the evolution of the intensity of the micro-PL spectrum of our system in the $T=$ $40-70 \mathrm{~K}$ range, wherein the effects of the coupling between the $\mathrm{X}$ line and the $\mathrm{PhC}$ cavity are most apparent. Up to a temperature of $50 \mathrm{~K}$, the overall micro-PL intensity undergoes a monotonic decrease, associated with the thermal activation of nonradiative recombination channels (as we will see in the following, the occurrence of this phenomenon [62] is also confirmed by our analysis of the QD recombination dynamics). For $T \geqslant 50 \mathrm{~K}$, as the $\mathrm{X}$ line is moved into resonance with the $\mathrm{CM}$, the enhancement of the radiative recombination rate due to the QD-cavity coupling-i.e., the Purcell effect [63] — results in a significant increase of the micro-PL intensity. At $T=60 \mathrm{~K}$, corresponding to near-zero detuning $(\Delta E \sim 0.6$ $\mathrm{meV}$ ), the micro-PL signal presents a relative maximum, after which the adverse effects of the increasing temperature on the QD emission take over, and the PL intensity again begins to decrease.

In parallel with the observation of the effects of varying $\Delta E$ on the overall micro-PL intensity, the analysis of the spectra displayed in Fig. 3 also allows us to investigate the evolution with temperature-and QD-CM coupling — of the linewidth of the $\mathrm{X}$ line. As summarized in the right inset of Fig. 3, the study of the temperature dependence of the FWHM of the $\mathrm{X}$ peak can provide us with a glimpse into the effects of the interaction of the QD exciton with its solid-state environment, most notably fluctuating charges-which contribute to the broadening of the $\mathrm{X}$ line via spectral diffusion/wandering [64-67] —and acoustic phonons-whose absorption and emission are known to result in the emergence of sidebands along the $X$ peak $[17,45,48,49,68-70]$. First of all, however, we need to discuss the origin of the relatively large linewidth observed, already at $T=10 \mathrm{~K}$, for the X peak of the QD investigated here. The QD in question was obviously not selected for its narrow linewidth, but rather because its $\mathrm{X}$ line was the closest to being in resonance with the $\mathrm{PhC}$ cavity mode. As such, its FWHM is relatively large when compared to the best QDs obtained in $\mathrm{H}$-irradiated $\mathrm{Ga}(\mathrm{AsN})$ - either by spatially selective hydrogenation [42] or by $\mathrm{H}$ removal [43] - which routinely reach linewidths in the $\sim 300-\mu \mathrm{eV}$ range. Of course, this is still quite high when compared to other techniques for the fabrication of site-controlled QDs, which have shown the ability to yield sub- $100-\mu \mathrm{eV}$ linewidths [25,71,72].

In our opinion, the origin of the sizable energy broadening measured in $\mathrm{Ga}(\mathrm{AsN}) / \mathrm{Ga}(\mathrm{AsN}): \mathrm{H}$ QDs at low $T$ is-at least - twofold. First of all, the relatively broad linewidth observed in these QDs is likely associated with the presence of large charge fluctuations in the $\mathrm{Ga}(\mathrm{AsN})$ matrix, due to the presence of localized states linked to single- $\mathrm{N}$ impurities and to $\mathrm{N}$ complexes. These charge fluctuations will be unavoidably associated with random oscillations of the QD energy, finally resulting in a significant linewidth broadening. Such charge-related effects are known to occur over timescales that span from $\sim 1$ ns-where they are usually referred to as spectral diffusion phenomena [73] — to the $\sim 100 \mathrm{~ms}-1 \mathrm{~s}$ range, where they lead to spectral wandering, i.e., energy fluctuations that can be detected with a conventional Si CCD (see, e.g., Ref. [32]).

In addition, it bears repeating that - as noted above-in the sample employed here the thickness of the capping GaAs layer grown on top of the $\mathrm{Ga}(\mathrm{AsN}) \mathrm{QW}$ was fixed at $30 \mathrm{~nm}$, in order to optimize the potential profile of the fabricated QD. Unfortunately, the need for a thin capping layer comes at the cost of keeping our $\mathrm{Ga}(\mathrm{AsN}) / \mathrm{Ga}(\mathrm{AsN}): \mathrm{H}$ QDs dangerously close to the sample surface, where the presence of a high concentration of dangling bonds - and of the accompanying fluctuating charges-is known to negatively affect the QD FWHM (see, e.g., Ref. [74]). In order to move the dot away from the surface - while retaining a sharp QD potential profile-in future iterations of this work we will aim at the fabrication of QDs based on $\mathrm{Ga}(\mathrm{AsN})$ epilayers, rather than QWs. Indeed, preliminary finite-element calculations of the $\mathrm{H}$ diffusion profile (see, e.g., Ref. [33]) suggest that the application of the masked-hydrogenation protocol to $\geqslant 100$ nm-thick Ga(AsN) samples would enable a better control of the QD depth and shape. By carefully adjusting the Ga(AsN) layer thickness and the mask size, this approach could lead to the realization of a QD in the center of the epilayer, as far as possible from the sample's interfaces. Moreover, it should be noted that this optimized fabrication protocol would also result in an improvement of the coupling between the QD and the relevant optical modes of the PhC membrane cavity (see below), since the electromagnetic field distribution of these modes is maximal in the center of the membrane.

Finally, it is also important to mention that QD broadening phenomena related to fluctuating charges are known to be sensitive to unconventional QD excitation schemes. Both resonant $p$-shell excitation [73] and two-photon excitation [75] have been reported to significantly reduce the QD FWHM and will certainly be tested in future developments of this work.

Coming to the temperature dependence of the FWHM of the QD—displayed in the right inset of Fig. 3-three separate regimes can be identified in the investigated $T$ (i.e., $\Delta E$ ) range: (1) For $T<30 \mathrm{~K}$, the FWHM is nearly independent of temperature. In this temperature range the sidebands associated with acoustic phonons are still weak when compared to the total intensity of the excitonic transition $[45,46,49]$, and-as already noted - the observed spectral broadening is chiefly due to charge-induced fluctuations of the energy of the $\mathrm{X}$ line $[64,67]$. (2) Between 30 and $60 \mathrm{~K}$, on the other hand, the role of phonons progressively becomes dominant, and the FWHM increases abruptly [68,69]. (3) For $T \geqslant 60 \mathrm{~K}$ (i.e., $\Delta E$ $<1 \mathrm{meV}$ ), finally, we observe a marked narrowing of the $\mathrm{X}$ line. While FWHM data in this range should be taken with the benefit of the doubt (in near-resonant conditions the spectrum effectively consists of a single peak, so that any attempt to fit it with two Lorentzians can lead to misleading results), this narrowing could be attributed to the interaction with the cavity mode, giving rise to phenomena similar to those discussed in Ref. [61]. In this respect, however, it is important to note that we do not see any concurrent change of the CM linewidth throughout the investigated temperature range, possibly due to the limited spectral resolution of our micro-PL setup $(\sim 300 \mu \mathrm{eV})$. 


\section{B. Modeling the dynamics of a QD weakly coupled with a PhC cavity}

As we will see in more detail below, the dissipative JaynesCummings model $[48,70,76,77]$ predicts a fairly simple dependence on $\Delta E$ for the effective recombination rate of a two-level system weakly coupled to a microcavity:

$$
\Gamma_{\text {tot }}=\gamma+\Gamma=\gamma+2 g^{2} \frac{\gamma_{\text {tot }}}{\gamma_{\text {tot }}^{2}+(\Delta \mathrm{E} / \hbar)^{2}},
$$

where $\gamma$ is the recombination rate of the exciton through channels other than the CM (nonradiative recombinations, leaky cavity modes, etc.) and $\Gamma$ is the rate at which excitons/photons transfer from the QD to the cavity and vice versa. In the expression for $\Gamma$ given in Eq. (1), $g$ is the X-CM coupling strength and $\gamma_{\text {tot }}$ is equal to

$$
\gamma_{\mathrm{tot}}=\left(\kappa+\gamma+2 \gamma_{\mathrm{dp}}\right) / 2 .
$$

$\kappa=\frac{E_{\mathrm{CM}}}{\hbar Q}\left(E_{\mathrm{CM}}\right.$ is the energy of the $\left.\mathrm{CM}\right)$ is the escape rate of a photon confined in the cavity, estimated at $760 \mathrm{~ns}^{-1}$ for the system investigated here. A Markovian pure dephasing rate, $\gamma_{\mathrm{dp}}$, is also included in Eq. (2), to account for the effects of the interaction of the QD exciton with the environment-once again, acoustic phonons $[17,18,45,48,49,70]$ and fluctuating charges [78-80]. In principle, it might be tempting to link $\gamma_{\mathrm{dp}}$ with the spectral broadening of the $\mathrm{X}$ line, given their common microscopic origin. In this respect, however, it must be noted that charge-induced fluctuations of the energy of the $\mathrm{X}$ line are commonly observed over timescales that can exceed the exciton lifetime by several orders of magnitude [64,67]; therefore, in the $T<30 \mathrm{~K}$ range [region 1 in the right inset of Fig. 3], in which spectral diffusion and/or wandering chiefly determine the X FWHM, the latter is likely to represent a gross overestimation of the actual contribution of the local charge environment to exciton dephasing. On the other hand, the effects of the interaction with acoustic phonons on QDmicrocavity coupling - which are likely dominant in the 30 $\mathrm{K} \leqslant T<60 \mathrm{~K}$ range, i.e., region 2 in the right inset of Fig. 3-are known to be reproduced rather poorly by a simple, Markovian dephasing term, thereby requiring an extension of the JC model (as discussed below and in Refs. [17,45,46,49]). For these reasons, in the remainder of this work we will use $\gamma_{\mathrm{dp}}=6 \mathrm{~ns}^{-1}$ (i.e., $4 \mu \mathrm{eV}$ ), as estimated in Ref. [48] by measuring the coherence of the single photons emitted by the QD with a Hong-Ou-Mandel interferometer. By picking this admittedly very low value, we choose to identify $\gamma_{\mathrm{dp}}$ as a baseline for the exciton dephasing rate; this will allow us to straightforwardly interpret the deviations of the experimental data from the predictions of the JC model in terms of the presence of additional dephasing channels in our system, either Markovian-due, e.g., to the interaction with fluctuating charges $[18,80]$ —or non-Markovian-i.e., associated with the absorption/emission of acoustic phonons [17,45,46,49].

The time-resolved experiments presented in this work were obtained with the same setup used for conventional micro-PL measurements, wherein the Si CCD detector was replaced with a $\mathrm{Si}$ avalanche photodiode (APD, time resolution $=300 \mathrm{ps}$ ). Additionally, we tested the ability of our coupled QD-PhC cavity system to emit single photons by measuring the second-order autocorrelation function of the (a) 1. e-h pairs reservoir 2. QD occupation states

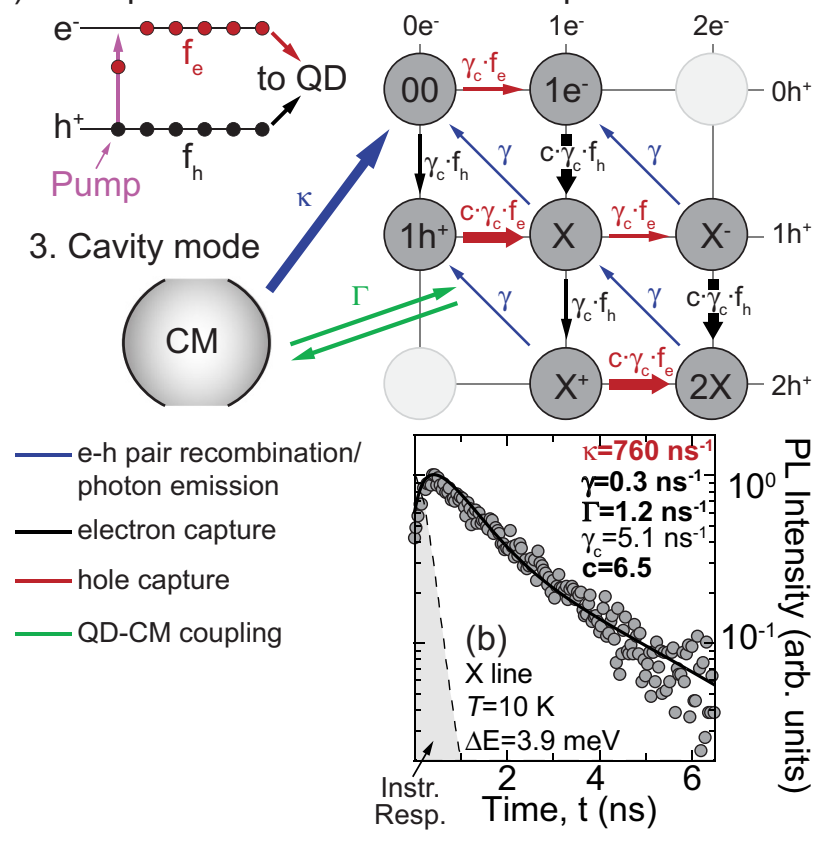

FIG. 4. (a) Schematic representation of the recombination dynamics of our QD-PhC cavity system (see main text and Appendix A). (b) Experimental time dependence (gray dots) of the micro-PL intensity of the $\mathrm{X}$ line $\left(T=10 \mathrm{~K}, P_{e x}=80 \mathrm{~W} / \mathrm{cm}^{2}\right)$. The instrumental response of the setup is also shown as a gray-shaded area, delimited by a dashed line. The experimental data are fitted by iteratively solving the system of rate equations discussed in the main text [see Eq. (4)], and the resulting curve is displayed as a thick black line. The fitting parameters obtained from this procedure are reported as follows: $\kappa$, which is fixed to $760 \mathrm{~ns}^{-1}$ (as estimated from the linewidth of the CM peak), is in bold red; $\gamma, \Gamma$, and $c$, which are going to be used as constants when fitting photon-correlation histograms (see Fig. 5), are in bold; $\gamma_{c}$ is in plain text.

$\mathrm{X}$ transition, $g_{\mathrm{X}-\mathrm{X}}^{(2)}(\tau)$. Photon correlation measurements were performed with a Hanbury Brown and Twiss interferometer [81]; i.e., the collected micro-PL signal was sent through a 50-50 beam splitter, dispersed with two independent $30-\mathrm{cm}$ monochromators (see description given above) and detected with two APDs (identical to the one used for time-resolved experiments), feeding a time correlator.

In order to extract quantitative information on the recombination dynamics of our system - that is, in order to estimate the parameters included in Eqs. (1) and (2) - the results of our time-resolved and photon correlation experiments were fitted with a rate-equation-based model, whose main features are sketched in Fig. 4(a). First of all [step 1 in Fig. 4(a)], a pulsed (deltalike) laser generates a reservoir of electron/hole pairs, acting as the sole source of carriers for the system. The probability of finding an electron, $f_{e}$, or a hole, $f_{h}$, in the reservoir is assumed to decay exponentially over time,

$$
f_{e, h}=e^{-\gamma_{\mathrm{c}}(t+\Delta \tau)},
$$

and the decay rate of the reservoir population is fixed to the rate of carrier capture by the $\mathrm{QD}, \gamma_{\mathrm{c}}$-which is also presumed to be the same for electrons and holes. Of course, the 
expression of $f_{e, h}$ given in Eq. (3) entails significant simplifications, which are discussed in greater detail in Appendix A. The inclusion of a time offset, $\Delta \tau$, however, crucially introduces the possibility to decouple the time at which we set the initial conditions for our rate-equation system $(t=0)$ from the time at which the excitation occurs $(t=-\Delta \tau)$. As discussed in Appendix B, this will prove to be a key ingredient in our analysis of photon correlation data.

Following photoexcitation, the carriers captured by the QD populate a ladder of occupation states-as sketched in step 2 of Fig. 4(a) - corresponding to an increasing number of electrons and holes in the QD [82]. Up to two electron-hole pairs (i.e., a biexciton, $2 \mathrm{X}$ ) can be accommodated in the dot; every possible configuration with $i=0,1,2$ electrons and $j=$ $0,1,2$ holes is taken into account, with the exception of the $2 e^{-}-0 h^{+}$and of the $0 e^{-}-2 h^{+}$cases, which are discounted on the basis of electrostatic considerations. Allowed transitions between different states are displayed as arrows, whose thickness is indicative of the transition rate. The system can "climb up" the states' ladder by capturing either an electron (horizontal red arrows) or a hole (vertical black arrows). To estimate the rate of each horizontal/vertical transition, the capture rate $\gamma_{\mathrm{c}}$ is weighted by the number of carriers available in the reservoir at a given moment in time $\left(f_{e / h}\right)$; moreover, the capture rate is enhanced (by a factor $c>1$ ) whenever carrier capture results in a neutral system (e.g., when going from a charged exciton to the biexciton state). Finally, QD states accommodating at least one $e-h$ pair can recombine-either radiatively or nonradiatively - with rate $\gamma$, assumed to be independent of the excitonic species (blue diagonal arrows).

It must be noted that the ladder of available QD states sketched in Fig. 4(a) is the simplest possible for a QD capable of hosting a biexciton. Given that semiconductor QDs-even in their simplest form, characterized by a single confined state for both electrons and holes-always have the ability to accommodate at least two $e-h$ pairs, the proposed model seems to be a fairly reasonable choice, capturing the main differences between real QDs and a simple two-level system without relying on an excessive number of parameters (we will return to this point later). Moreover-as detailed in Appendix A-some of the unique features of our QD-PhC cavity system still allow for a simplified treatment of the effects of the integration of the dot in an optical microcavity. Owing to the fact that (i) only the $\mathrm{X}$ transition is coupled to the CM and that (ii) we are still within the weak-coupling regime, indeed, the fundamental cavity mode can be introduced in our model as an extra occupation state [see step 3 of Fig. 4(a)], i.e., as a single additional rate equation in the set describing the time evolution of the QD-PhC cavity system. Under such conditions, the time evolution of the system can be obtained by solving the following set of rate equations:

$$
\begin{aligned}
\frac{d p_{00}}{d t} & =-\left(2 \gamma_{\mathrm{c}} f_{e, h}\right) p_{00}+\gamma p_{11}+\kappa p_{\mathrm{CM}}, \\
\frac{d p_{10}}{d t} & =\left(\gamma_{\mathrm{c}} f_{e, h}\right) p_{00}-\left(c \gamma_{\mathrm{c}} f_{e, h}\right) p_{10}+\gamma p_{21}, \\
\frac{d p_{01}}{d t} & =\left(\gamma_{\mathrm{c}} f_{e, h}\right) p_{00}-\left(c \gamma_{\mathrm{c}} f_{e, h}\right) p_{01}+\gamma p_{12},
\end{aligned}
$$

$$
\begin{aligned}
\frac{d p_{11}}{d t}= & -\left(\gamma+\Gamma+2 \gamma_{\mathrm{c}} f_{e, h}\right) p_{11}+\left(c \gamma_{\mathrm{c}} f_{e, h}\right)\left(p_{10}+p_{01}\right) \\
& +\gamma p_{22}+\Gamma p_{\mathrm{CM}} \\
\frac{d p_{21}}{d t}= & \left(\gamma_{\mathrm{c}} f_{e, h}\right) p_{11}-\left(\gamma+c \gamma_{\mathrm{c}} f_{e, h}\right) p_{21} \\
\frac{d p_{12}}{d t}= & \left(\gamma_{\mathrm{c}} f_{e, h}\right) p_{11}-\left(\gamma+c \gamma_{\mathrm{c}} f_{e, h}\right) p_{12} \\
\frac{d p_{22}}{d t}= & \left(c \gamma_{\mathrm{c}} f_{e, h}\right)\left(p_{21}+p_{12}\right)-\gamma p_{22} \\
\frac{d p_{\mathrm{CM}}}{d t}= & \Gamma p_{11}-(\kappa+\Gamma) p_{\mathrm{CM}}
\end{aligned}
$$

wherein the time dependence of $f_{e, h}(t)$ has been omitted for brevity. Here, $p_{i j}$ is the probability of finding the system in the QD state with $i$ electrons and $j$ holes, while $p_{\mathrm{CM}}$ is the probability that a photon populates the CM (it should be noted that $\frac{d p_{\mathrm{CM}}}{d t}+\sum_{i, j} \frac{d p_{i j}}{d t}=0$, consistent with the condition $p_{\text {tot }}=$ $\left.p_{\mathrm{CM}}+\sum_{i, j} p_{i j}=1\right)$. As already mentioned, the $\mathrm{CM}$ is treated as an additional occupation state, which is only populated via transfer from the $\mathrm{X}$ state.

In principle, the time evolution of the probabilities found by solving Eq. (4) should closely match that of the microPL intensity of the corresponding QD transition, provided that (i) the appropriate set of initial conditions is employed and (ii) we can infer reasonable estimates for the unknown parameters present in Eq. (4). As far as the initial conditions are concerned we have already noted that, for time-resolved micro-PL measurements, the time at which the laser pulse creates a finite population of electron-hole pairs in the reservoir coincides with $t=0$ [that is, the time delay $\Delta \tau$-as defined in Eq. (3) -is set to 0]. In the absence of other excitation sources, then, the QD-PhC cavity system must be in its empty state at $t=0$; i.e., $p_{00}(t=0)=1$.

On the other hand, a total of six parameters is included in Eq. (4) [after accounting for $\Delta \tau$, which is not explicitly present in Eq. (4) but is contained in the definition of $f_{e, h}(t)$ provided in Eq. (3)]. Their physical meaning has been discussed in the previous paragraphs, and a visual representation of their role in determining the dynamics of our integrated QD-PhC cavity system is provided in Fig. 4(a). As mentioned above, $\Delta \tau$ can be set to zero when dealing with time-resolved micro-PL data, while $\kappa$ can be fixed to $760 \mathrm{~ns}^{-1}$ - the value resulting from the linewidth of the CM peak-for the microcavity investigated here. The values of the remaining parameters can be estimated by fitting the probabilities $p_{i j}\left(p_{\mathrm{CM}}\right)-$ obtained by numerically solving Eq. (4) - to the measured time dependence of the micro-PL intensity of the $i j$-th QD transition (of the CM line). The instrumental response of our time-resolved setup is taken into account by convolving it with $p_{i j}\left(p_{\mathrm{CM}}\right)$ prior to fitting. An example of such a fit-relative to the $\mathrm{X}$ transition of our QD-PhC cavity system, at $T=$ $10 \mathrm{~K}$-is displayed in Fig. 4(b), together with the instrumental response of the setup; the estimates attained for $\gamma_{\mathrm{c}}, c, \gamma$, and $\Gamma$ are all reported in the figure. It is worth noting that the value obtained for $c, 6.5$, is indeed greater than 1 , as expected from its definition (see above). Most importantly, however, the fitting procedure outlined here yields reliable estimates for the values of $\gamma$ and $\Gamma$, thus providing direct, quantitative 


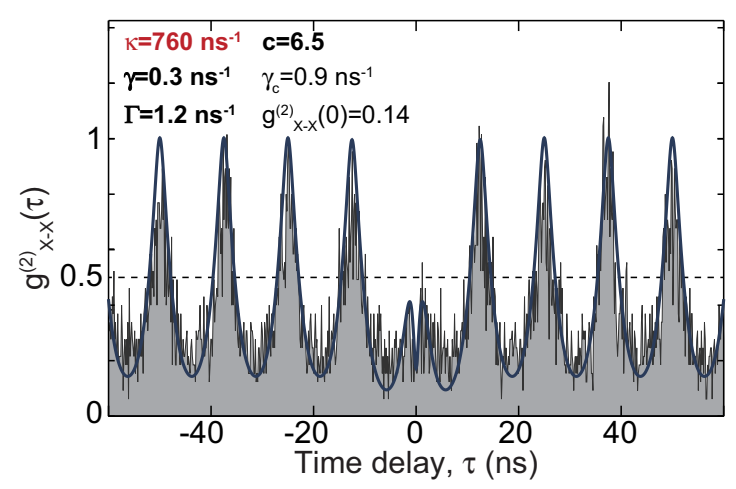

FIG. 5. Second-order autocorrelation histogram, $g_{\mathrm{X}-\mathrm{X}}^{(2)}(\tau)$, of the $\mathrm{X}$ transition of the investigated QD-PhC cavity system $(T=10 \mathrm{~K}$, $P_{e x}=30 \mathrm{~W} / \mathrm{cm}^{2}$, histogram bin size $=150 \mathrm{ps}$ ). The thick blue line is a fit to the experimental data (displayed as a gray-shaded area plot), obtained as described in Appendix B.

information on the radiative recombination dynamics of our QD-PhC cavity system.

\section{Photon correlation}

The reliability of the values of $\gamma$ and $\Gamma$ obtained from timeresolved data is further confirmed by the possibility of treating them as constants when using our model to fit photon correlation measurements performed on the same system (see Fig. 5). In order to define the fitting procedure best suited for this type of data, we need to define the relationship between $g_{i j-k l}^{(2)}(\tau)$ the second-order correlation function between two arbitrary QD transitions, $i j$ and $k l$-and the solutions to Eq. (4), i.e., the $p_{k l}(\tau)$ probabilities. As detailed in Appendix B, this can be done by extending the considerations made in Ref. [82] to the case of pulsed excitation.

Notably, the experimental data shown in Fig. 5-which refer to the $i j=k l=11$ case, i.e., to the autocorrelation of the exciton-are reproduced very well by the fitted curvebased on Eq. (B3); see Appendix B-a fact that is all the more remarkable when considering that such an agreement was obtained with a single fitting parameter, $\gamma_{\mathrm{c}}$. As mentioned above, indeed, $\gamma$ and $\Gamma$-as well as $c$-were fixed to the values obtained from fitting the time-resolved microPL measurements presented in Fig. 4(b), also relative to the $\mathrm{X}$ transition and obtained under very similar experimental conditions. The different values of $\gamma_{c}$ obtained from the fits displayed in Figs. 4(b) and $5\left(5.1 \mathrm{~ns}^{-1}\right.$ from time-resolved micro-PL, $0.9 \mathrm{~ns}^{-1}$ from autocorrelation measurements), on the other hand, are probably reflective of the approximations we made when defining $f_{e, h}(t)$. As discussed at length in Appendix A, indeed, the time dependence of the population of our reservoir of electrons and holes might be considerably more complex than a single exponential function [as we postulated when defining Eq. (3)], and in any case the decay rate of the reservoir population might differ significantly from $\gamma_{\mathrm{c}}$, the QD capture rate. As a consequence, different effective values of $\gamma_{c}$ might be needed to fit the results of different experiments, which probe separate aspects of the dynamics of our QD-PhC cavity system. Indeed, time-resolved (TR) micro-PL histograms are constructed by plotting the number of detected photons as a function of their time delay with respect to the excitation laser pulse; as such, all radiative recombinations associated with a given QD state-in our case, the exciton-contribute to the signal, regardless of when they occur. As a consequence, the value of $\gamma_{c}$ estimated by fitting TR micro-PL data likely represents a reasonable estimate of the average carrier capture rate of the QD. On the other hand, for exciton autocorrelation histograms the presence of a finite number of counts around a near-zero time delay $(\tau \sim 0)$ is associated with excitons that recombine only after a first exciton has already been detected, i.e., on average, relatively late with respect to the excitation pulse. The estimate of the capture rate obtained from fitting this type of data is therefore much more sensitive to the eventual presence of long-lived carriers in the reservoir feeding the QD, thus resulting in a smaller value of $\gamma_{\mathrm{c}}$.

Aside from allowing us to confirm the general validity of our model, the analysis of pulsed autocorrelation measurements also provides us with a useful benchmark for the performance of our nanodevices as triggered single-photon emitters. If, indeed, a single photon is generated for each pulse, the probability of observing a second exciton immediately after the detection of the first one is null by definition, and we should strictly observe $g_{\mathrm{X}-\mathrm{X}}^{(2)}(\tau=0)=0$. In practical terms, however, the generally accepted criterion for identifying a single-photon emitter is that $g_{\mathrm{X}-\mathrm{X}}^{(2)}(0)<0.5$, which stems from combining the well-known identity, $g_{N-N}^{(2)}(\tau=0)=1-\frac{1}{N}-$ valid for the $N$ th eigenstate of the photon number operator [83] — with the condition that $N$ be smaller than 2. As shown in Fig. 5(a), our system easily clears this bar, as $g_{\mathrm{X}-\mathrm{X}}^{(2)}(0)<0.2$ $(=0.14$, according to our fit). If we extend our analysis to finite time delays, it becomes clear that-as accounted for and correctly reproduced by our model-there is a nonzero probability of observing two photons per pulse, due to the capture of a second $e$ - $h$ pair by the QD following the recombination of the first exciton. However, it must be noted that $g_{\mathrm{X}-\mathrm{X}}^{(2)}(\tau)$ stays well below 0.5 across the entire central peak, ultimately confirming the ability of $\mathrm{Ga}(\mathrm{AsN}) / \mathrm{Ga}(\mathrm{AsN}): \mathrm{H}$ QDs to emit single photons even after their integration with a $\mathrm{PhC}$ cavity.

\section{Effects of QD-PhC cavity coupling on the exciton dynamics}

Having demonstrated our ability to deterministically integrate a single-photon emitter in a $\mathrm{PhC}$ microcavity, we can now move on to the investigation of the more subtle effects of such integration on the QD dynamics. As shown in Fig. 6(a), the transient behavior of the micro-PL signal associated with the $\mathrm{X}$ transition varies sharply as the QD is moved into resonance with the $\mathrm{CM}$, with the clear emergence of a fasterdecaying component for decreasing QD-CM energy detuning, $\Delta E$. Aside from being a clear manifestation of the Purcell effect [63], such departure from a monoexponential time dependence is clearly linked to the progressive opening of an additional recombination channel for the QD, due to the increasing strength of the interaction between the $\mathrm{X}$ transition and the fundamental mode of the cavity. As $\Delta E$ is reduced, indeed, the transfer of photons to the $\mathrm{CM}$ - which occurs with rate $\Gamma$; see Eq. (1) — becomes increasingly efficient, immediately leading to the emission of light into the environment, due to the short lifetime $\left(\kappa=760 \mathrm{~ns}^{-1}\right)$ of the cavity photons. As 


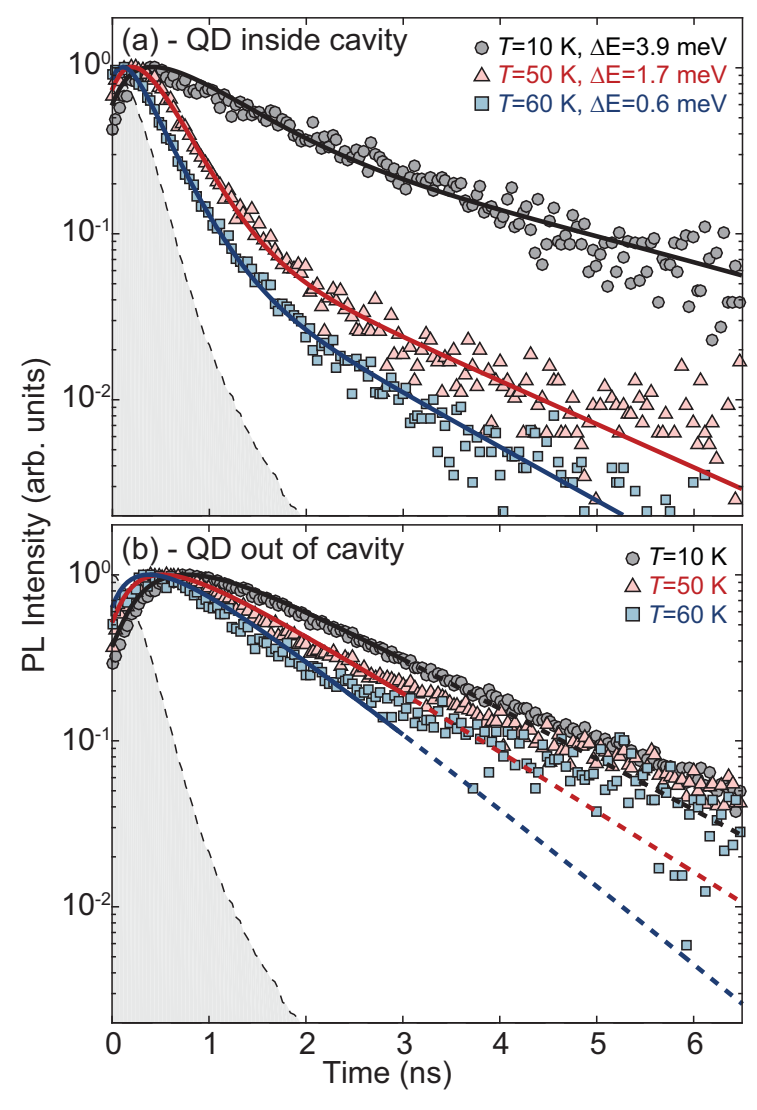

FIG. 6. (a) Time dependence of the micro-PL intensity of the $\mathrm{X}$ transition in the investigated QD-PhC cavity system, plotted for different temperatures, i.e., different values of $\Delta E$. The experimental data acquired at $T=10,50$, and $60 \mathrm{~K}$ are shown as gray dots, light red triangles, and light blue squares, respectively. The thick continuous lines (black for $T=10 \mathrm{~K}$, red for $50 \mathrm{~K}$, and blue for $T=$ $60 \mathrm{~K}$ ) are fits to the data based on the model sketched in Fig. 4(a) and discussed in the main text and in Appendix A. The instrumental response of our time-resolved micro-PL setup is also shown as a gray-shaded area, delimited by a dashed line. (b) As in (a) but for a "bare" (i.e., not integrated with a PhC cavity) QD. The thick lines are fits based on the same model used for the in-cavity QDs, but with the QD-cavity coupling switched off (i.e., $\Gamma$ is set equal to zero). For times $t>3$ ns the experimental data deviate significantly from the fit (displayed as dashed lines from this point onward), due to the onset of a biexponential behavior associated with features of the QD dynamics that are not taken into account by our simplified QD model (see main text).

a result, the integration with the $\mathrm{PhC}$ cavity turns out to be the defining factor for the QD dynamics, introducing a dichotomy between the only state that is efficiently coupled to the cavity mode- the single exciton, which can emit photons through the $\mathrm{CM}$ and has an effective recombination rate approximately equal to $\Gamma_{\text {tot }}=\gamma+\Gamma$ - and all the other, uncoupled QD states, for which spontaneous emission is severely inhibited. The presence of these "longer-lived" states crucially affects the exciton dynamics, leading to the emergence of the slow component characterizing the biexponential behavior of the $\mathrm{X}$ transition. Moreover, for the purposes of our model, the inhibition of spontaneous emission for all the uncoupled QD states results in the possibility to set all their recombination rates to $\gamma$. This is a very significant simplification, which allows us to accurately describe the QD dynamics with a limited number of parameters (four, as discussed above).

The influence of the cavity's presence on the time evolution of the intensity of the $\mathrm{X}$ line is confirmed by the comparison of the TR micro-PL data of our integrated QD-PhC cavity system [see Fig. 6(a)] with Fig. 6(b), displaying how the exciton dynamics varies with temperature for a "bare" (i.e., not integrated with a PhC cavity) QD. It should be noted that the investigated bare QD is embedded in a released GaAs membrane (see, e.g., Ref. [43]), so that its local environment is as similar as possible to that of in-cavity QDs (except, of course, for the presence of the PhC lattice). Within the investigated temperature range $(10-60 \mathrm{~K})$, which matches the one displayed in Fig. 6(a) (let us remind the reader that the QD is tuned into resonance with the $\mathrm{CM}$ by changing the temperature; see Fig. 3) there is only a minor speed-up in the QD exciton dynamics. As briefly anticipated when discussing Fig. 3-and commonly observed in semiconductor QDs; see, e.g., Ref. [84] - this effect is likely due to the progressive activation of nonradiative recombination channels as the temperature is increased [62]. However, while undoubtedly present, such temperature-related increase of the radiative recombination rate is clearly negligible with respect to the effects of QD-cavity coupling. This is already obvious from a visual inspection of the experimental results, which evidence a much stronger dependence on temperature-i.e., on QD-cavity energy detuning, $\Delta E$-for the dynamics of the in-cavity QD with respect to the out-of-cavity case; however, the same conclusions are borne out by a more quantitative analysis, carried out by fitting the data reported in Figs. 6(a) and 6(b) with our model. When applied to our integrated QD$\mathrm{PhC}$ cavity system, such fitting procedure yields the curves displayed as thick continuous lines in Fig. 6(a), while also allowing us to estimate the values of $\gamma$ and $\Gamma$ for the different time transients investigated. As reported in Fig. 7 (red diamonds), the effective recombination rate, $\Gamma_{\text {tot }}=\gamma+\Gamma$, varies by nearly one order of magnitude in the investigated detuning range, i.e., for temperatures varying between 10 and $60 \mathrm{~K}$. This is in stark contrast with the trend observed for $\gamma_{0}$, the recombination rate of the bare $\mathrm{QD}$, which only decreases by $\sim 50 \%$ within the same $T$ interval (also reported, as blue squares, in Fig. 7). As a matter of fact, the behavior of $\gamma_{0}$ with temperature resembles much more closely that of $\gamma$ (white diamonds in Fig. 7), the recombination rate through channels other than the CM (see above); this is not surprising, given that nonradiative channels play a crucial role in the determination of both rates [62,79].

As regards the values of $\gamma_{0}$ reported in Fig. 7, we must mention that they were extracted from a simplified version of our rate-equations model, wherein the coupling with the cavity was "turned off;" i.e., $\Gamma$ was set to zero. Given that we kept using a single recombination rate for all excitonic states (that is, precisely $\gamma_{0}$ ), such simplified model is not able to follow the biexponential behavior of the QD dynamicswhich, albeit reduced with respect to the in-cavity case, is also present in the TR signal of bare QDs [see Fig. 6(b)]. Such biexponential behavior, which in $\mathrm{Ga}(\mathrm{AsN}) / \mathrm{Ga}(\mathrm{AsN}): \mathrm{H}$ nanostructures was observed in Ref. [42], is indeed typical of semiconductor QDs. Depending on the specific system, it 


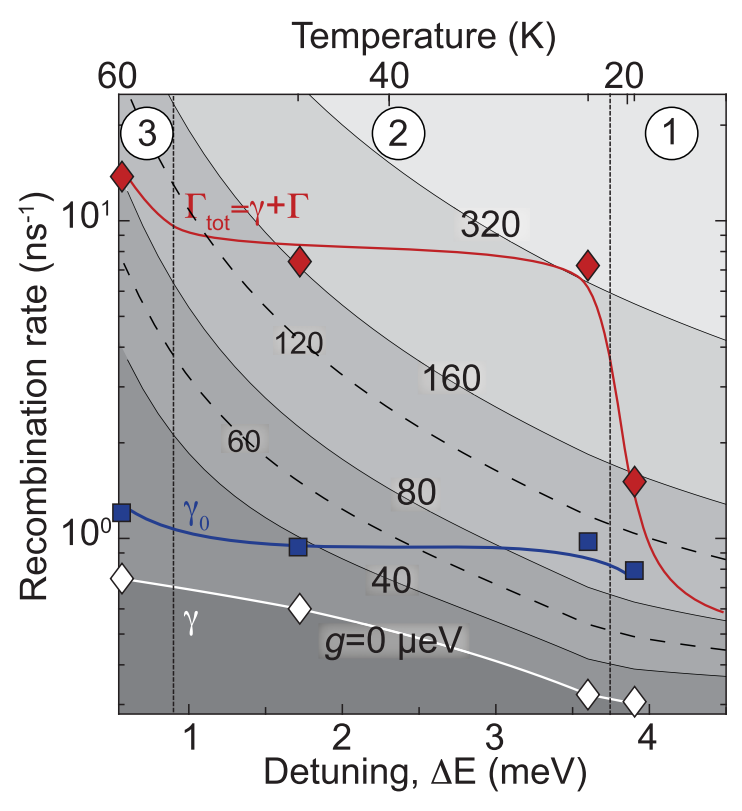

FIG. 7. The total recombination rate of the exciton of our integrated QD-PhC cavity system, $\Gamma_{\text {tot }}=\gamma+\Gamma$, is plotted as a function of $\Delta E$ (red diamonds; the red line is a guide to the eye). The plot is divided into three regions [labeled as 1-3], corresponding to the temperature (i.e., detuning) intervals defined in the right inset of Fig. 3. A contour plot of the theoretical dependence of $\Gamma_{\text {tot }}$ on $\Delta E$, computed for different values of the coupling strength $g$ and based on the Jaynes-Cummings model, is included for comparison (in shades of gray; the contour lines corresponding to the range $g=60-120 \mu \mathrm{eV}$ are displayed as dashed lines). The values of $\gamma$, the recombination rate due to nonradiative processes, recombination through leaky cavity modes, etc., are also shown as white diamonds. We also report (as blue squares; the blue line is a guide to the eye) the values of $\gamma_{0}$, the recombination rate of a "bare," out-of-cavity QD, measured at the temperatures corresponding to the reported energy detuning values $(10,30,50,60 \mathrm{~K})$

could be due to relaxation phenomena involving the excited states of the QD [85,86], to the presence of carrier traps in the QD surroundings [87], to dark exciton-related processes $[88,89]$, and possibly to all (or some) of the above. Clearly, including one or more of these mechanisms would make the model considerably more cumbersome. While this would be a necessity if our goal were an in-depth analysis of the TR micro-PL signal of bare $\mathrm{Ga}(\mathrm{AsN}) / \mathrm{Ga}(\mathrm{AsN}): \mathrm{H}$ QDs, there is no need for such complications in the present work, chiefly aimed at the quantification of the effects of QD-PhC cavity integration on the exciton dynamics. In any case, it should be noted that the fitting curves reported in Fig. 6(b) closely match the experimental data for $t \geqslant 3 \mathrm{~ns}$, so that the obtained value of $\gamma_{0}$ is actually a good estimate of the higher recombination rate (i.e., shorter decay time) characterizing the initial phase of the exciton decay.

Coming back to the discussion of the data displayed in Fig. 7, it can be interesting to compare the experimental values of $\Gamma_{\text {tot }}=\gamma+\Gamma$ with the dependence of $\Gamma_{\text {tot }}$ on $\Delta E$ predicted by the JC model for a two-level system coupled to a cavity. The contour plot in the background of Fig. 7 was indeed obtained by using Eq. (1) to compute $\Gamma_{\text {tot }}$ for values of the coupling strength $g$ ranging between 0 and $320 \mu \mathrm{eV}$. This range is centered around the maximal coupling expected for a $\mathrm{Ga}(\mathrm{AsN}) / \mathrm{Ga}(\mathrm{AsN}): \mathrm{H}$ QD perfectly aligned with the fundamental mode of an $L_{3}$ cavity, $g_{\max }=160 \mu \mathrm{eV}$. This value can be easily obtained from the equation [76]

$$
g_{\max }=\frac{1}{4 \pi} \sqrt{\frac{3 \gamma_{0} \omega}{V}}
$$

wherein we used $\gamma_{0} \sim 1 \mathrm{~ns}^{-1}$, as estimated by averaging the values obtained for several bare $\mathrm{Ga}(\mathrm{AsN}) / \mathrm{Ga}(\mathrm{AsN}): \mathrm{H}$ QDs [see Fig. 6(b), as well as Ref. [42]]. The effective volume of the CM, $V$, is $\sim 0.65\left(\frac{\lambda}{n}\right)^{3}$ for the fundamental mode of an $L_{3}$ cavity (see, e.g., Ref. [90]).

The other parameter included in Eq. (1), $\gamma_{\text {tot }}$ [see Eq. (2)], can be evaluated by setting $\kappa$ to $760 \mathrm{~ns}^{-1}$-as obtained from the CM linewidth-and $\gamma_{\mathrm{dp}}$ to $6 \mathrm{~ns}^{-1}$ (i.e., $4 \mu \mathrm{eV}$ [48]; see above). For $\gamma$, we used an interpolation of the values obtained from the fits reported in Fig 6(a); however, it must be mentioned that the contribution to $\gamma_{\text {tot }}$ of both $\gamma$ and $\gamma_{\mathrm{dp}}$ is virtually negligible, given the much higher value taken by $\kappa$ for our QD-PhC cavity system. As noted above, the slight decrease observed in the values of $\gamma$ (shown as white diamonds in Fig. 7) as a function of increasing $\Delta E$ is-akin to the trend observed for $\gamma_{0}$-due to the progressive deactivation of nonradiative recombination channels as the temperature is decreased.

As Fig. 7 clearly shows, the integration with a PhC cavity deeply alters the recombination dynamics of our sitecontrolled QDs, with a maximal effective recombination rate-obviously obtained for the lowest detuning investigated here, $\Delta E=0.6 \mathrm{meV}$ - equal to $\Gamma_{\text {tot }}=14 \mathrm{~ns}^{-1}$. This value, which is more than 10 times higher than the recombination rates typically measured for bare $\mathrm{Ga}(\mathrm{AsN}) / \mathrm{Ga}(\mathrm{AsN}): \mathrm{H}$ QDs $\left[\sim 1 \mathrm{~ns}^{-1}\right.$ or lower; see Fig. 6(b)], is in very good agreement with the Purcell enhancement predicted by the JC model for $g=80 \mu \mathrm{eV}$ (see contour plot in Fig. 7). Such a value of $g$, while unmistakably lower than $g_{\max }$, is consistent with the coupling strength that one could reasonably expect for our QD-PhC system, given that the fabricated $\mathrm{Ga}(\mathrm{AsN}) / \mathrm{Ga}(\mathrm{AsN})$ : $\mathrm{H}$ QDs are vertically misaligned (by $\sim 50 \mathrm{~nm}$ ) from the center of the cavity, due to the asymmetric positioning of the as-grown $\mathrm{Ga}(\mathrm{AsN}) \mathrm{QW}$ within the membrane (as discussed in Sec. II A). In order to account for the uncertainty in the determination of the coupling strength from the comparison between the experimental data and the expectations of the JC model, the contour lines corresponding to the range $g=60-120 \mu \mathrm{eV}$ are also displayed (as dashed lines) in Fig. 7.

Interestingly, the experimental results reported in Fig. 7 deviate significantly from the behavior predicted by JC theory as we move away from near-zero detuning. In the $\Delta E \sim$ $1.5-3.5 \mathrm{meV}$ detuning range, in particular, the values of $\Gamma_{\text {tot }}$ obtained for our QD-PhC cavity system are much higher than the expectations of the JC model for any realistic value of $g$. As indicated in the figure, this detuning range corresponds to the temperature interval $(30 \mathrm{~K}<T<60 \mathrm{~K}$ ) labeled as region 2 in the right inset of Fig. 3, wherein exciton dephasing processes are primarily due to the interaction with acoustic phonons. As noted above, in this regime the spectral lines 
associated with radiative QD transitions are typically broadened by the presence of sidebands associated with phonon emission/absorption, which extend over few $(\leqslant 5) \mathrm{meV}$ and are known to effectively mediate the coupling between the QD and a moderately detuned CM [17,18,45,49]. In order to correctly account for the role of phonons in the determination of the detuning-dependent QD-CM coupling, indeed, Eq. (1) must be modified as follows $[46,49]$ :

$$
\Gamma_{\text {tot }}^{\text {phon. }}=\gamma+2 g^{2} \frac{\gamma_{\text {tot }}}{\gamma_{\text {tot }}^{2}+(\Delta E / \hbar)^{2}}\left[1+\frac{1}{\hbar^{2} \gamma_{\text {tot }}} \Phi(\Delta E, T)\right],
$$

where $\Phi(\Delta E, T)$ is defined as an effective phonon density of states. As verified both theoretically and experimentally in Ref. [49], this quantity is zero for $\Delta E=0$-where Eq. (6) is indeed identical to Eq. (1), and the Purcell enhancement is in line with the expectations of the "phononless" JC model, as already discussed-and has a maximum in the $|\Delta E| \leqslant 5 \mathrm{meV}$ range, obviously resulting in an additional enhancement of the $\mathrm{QD}$ radiative recombination rate within the same interval. The data shown in Fig. 7 are in excellent qualitative agreement with this picture, even though more systematic measurements will be required in order to fully characterize $\Phi(\Delta E, T)$ for our QD-PhC cavity system.

As already noted in the Introduction, the mere ability to discern the role of phonons in the determination of the QD-CM coupling strength points out one of the inherent advantages of $\mathrm{Ga}(\mathrm{AsN}) / \mathrm{Ga}(\mathrm{AsN}): \mathrm{H}$ QDs for the realization of cavity-QED experiments. As noted by several reports [78,91], indeed, integrated QD-PhC cavity systems based on self-assembled quantum dots typically continue to feature a detectable CM peak in their emission spectra, even when the CM energy is out of resonance with the closest QD transition by more than 100 times the mode linewidth (i.e., $\gg 10 \mathrm{meV}$ ). This observation, which is impossible to reconcile with JC theory, can only be explained by invoking a fairly complex mechanism of cavity feeding [78]. According to this explanation, for large QD-CM detuning the cavity is fed through a quasicontinuum of transitions, associated with the hybridization of the multiexcitonic levels of the QD with the surrounding wetting layer (WL). The presence of such a quasicontinuum in the spectrum of self-assembled QDs is a major deviation from the "artificial atom" picture, and it prevents the establishment of true quantum strong coupling (i.e., strong coupling at the level of single quanta $[13,92,93]$ ) between the QD and the CM. The situation improves dramatically in QD systems for which the WL is very thin or absent, as reported in Ref. [17] for site-controlled (InGa)As dots grown in lithographically defined tetrahedral recesses. Thanks to the inherent lack of a WL, such "far off-resonance" coupling is greatly diminished in $\mathrm{Ga}(\mathrm{AsN}) / \mathrm{Ga}(\mathrm{AsN}): \mathrm{H}$ QDs, which can thereby be regarded as being a better approximation of an "artificial atom in the solid state" than their self-assembled Stranski-Krastanov counterparts.

\section{CONCLUSIONS}

In summary and conclusion, we demonstrated the ability to deterministically pair a $\mathrm{PhC}$ microcavity with the single, site- controlled QDs fabricated by spatially selective $\mathrm{H}$ irradiation of $\mathrm{Ga}(\mathrm{AsN})$. After integration with the cavity, these QDs fully retain their ability to emit triggered single photons; moreover, the effects of the modified electromagnetic environment on the QD properties are clearly visible in the time dependence of the micro-PL signal associated with the exciton transition, which undergoes sharp changes as the QD-PhC energy detuning, $\Delta E$, is progressively reduced.

By fitting the experimental data with a theoretical model based on a system of rate equations, we can extract the effective recombination rate of the QD exciton, $\Gamma_{\text {tot }}$, which can then be compared with the expectations of dissipative JaynesCummings theory. While for $\Delta E \sim 0$ the measured value of $\Gamma_{\text {tot }}$ is in good agreement with the predictions of the JC model, in an intermediate detuning range $(\Delta E \sim 1.5-3.5 \mathrm{meV})$ the interaction of the QDs with acoustic phonons effectively mediates the QD-PhC cavity coupling, resulting in an additional enhancement of the radiative recombination rate of the dot. The observation of this important effect of the solid-state environment on the dot properties is a strong indication of the truly zero-dimensional nature of $\mathrm{Ga}(\mathrm{AsN}) / \mathrm{Ga}(\mathrm{AsN}): \mathrm{H}$ QDs, which greatly bolsters their status as promising candidates for the implementation of cavity QED-based experiments and applications. As already mentioned in the Introduction, indeed, these QDs could be easily employed as sources of nonclassical light in complex photonic circuits [11], embedding all the elements required for the on-chip generation, guiding/splitting [94,95], and detection [96] of single photons.

Within this context, it is important to correctly gauge the importance — both fundamental and technological —of the observation that the interaction with phonons significantly extends (up to $\sim \pm 3 \mathrm{meV}$; see Fig. 7) the coupling range between $\mathrm{Ga}(\mathrm{AsN}) / \mathrm{Ga}(\mathrm{AsN})$ :H-based quantum emitters and an optical microcavity. On the one hand, indeed, such broadband coupling may partially lift the requirement of a very precise spectral matching (within less than $\sim 1 \mathrm{meV}$, according to an estimate based on the "standard" JC model [22]) between all the emitters embedded within a device and the cavity mode. On the other hand, interactions with acoustic phonons-as well as with fluctuating charges - are known to be responsible for the dephasing phenomena associated with the less than perfect $(<100 \%)$ degree of indistinguishability measured for the single photons emitted by QDs embedded in a solid-state matrix. While the effect of fluctuating charges is (i) typically occurring over longer timescales [73], and (ii) can be limited by resonant excitation schemes [75], phonon dephasing is more difficult to eliminate completely. Furthermore, as already noted, the energy gains/losses associated with the interaction with phonons lead to the emergence of sidebands in the exciton spectrum, funneling photons away from the zero-phonon line (ZPL) and thus reducing the source efficiency [97-99]. It is therefore reasonable to wonder whether a scheme that relies on such phonon sidebands (PSBs) to extend the energy range of the interaction between the QD and the PhC cavity would be able to meet the benchmarks required by quantum information and communications applications.

In our opinion, at least four considerations can be made to assuage these concerns. First of all, recent experiments $[99,100]$ and theoretical calculations [98] suggested that 
phonon-induced decoherence may be significantly reduced by coupling the ZPL of the emitter with a microcavity, theoretically reaching $>99 \%$ indistinguishability-and $\sim 96 \%$ source efficiency - under resonant conditions. Even though a dropoff in performance-especially in terms of brightness-is to be expected if the PSBs, rather than the ZPL, are involved in the coupling, this approach is certainly worth pursuing further. Also, many applications in short-distance quantum communications, quantum cryptography, or quantum metrology do not actually require highly indistinguishable photons-as also noted in Ref. [97], which first highlighted the potential role of the phononic environment in extending the range of lightmatter interaction in the solid state.

Second, the emission of entangled photons-one of the most relevant applications for QDs in the field of quantumlight generation-is mostly unaffected by phonon-induced dephasing, as first noted in Ref. [101] and then confirmed by several other reports; see, e.g., Ref. [75].

Third, a striking confirmation of the possibility of exploiting exciton-phonon interactions to efficiently generate indistinguishable, highly entangled photons has been recently provided in Ref. [102]. In that work, Reindl et al. demonstrated a near-unity fidelity of entanglement and, perhaps even more impressively, a high degree of indistinguishability for the photons generated by a QD excited through a novel quasiresonant two-photon scheme, in which phonon sidebands play a crucial role. In the reported experiment, indeed, the laser was detuned by $\sim 350 \mu \mathrm{eV}$ from the resonant condition for two-photon excitation; i.e., it was nearly aligned with the maximum of the acoustic PSB. Most notably, this quasiresonant excitation scheme could be employed to generate indistinguishable photons from two distant QDs, in an intrinsically more robust fashion with respect to current stateof-the-art schemes requiring perfectly resonant excitation for the two QDs. This experiment beautifully illustrates how, in solid-state systems, the interaction with phonons can significantly relax the otherwise very strict constraints required for the observation of resonant phenomena, making them much less dependent on extrinsic, difficult-to-control variables (disorder, charge fluctuations, etc.) and, thus, potentially easier to exploit for applications operating outside of a controlled lab environment.

Last but not least, it should not be forgotten that the present work deals, perhaps mainly, with the successful demonstration of a method to deterministically integrate site-controlled $\mathrm{Ga}(\mathrm{AsN}) / \mathrm{Ga}(\mathrm{AsN}): \mathrm{H}$ QDs with photonic crystal cavities. The natural landing point of this method is the realization of photonic structures embedding more than one nanoemitter within the same device. Many of the technologically and scientifically relevant applications that could be realized with this method do not require indistinguishable photons to function optimally: For example, the simultaneous positioning of a fixed number of $\mathrm{Ga}(\mathrm{AsN}) / \mathrm{Ga}(\mathrm{AsN}): \mathrm{H}$ QDs in a $\mathrm{PhC}$ cavity might enable the observation of cavity-mediated coupling between two or more spatially distant QDs [103]—possibly paving the way to the realization of QD solids [104] —as well as the realization of low-threshold, few-QD PhC nanolasers [105], wherein all the embedded QDs are optimally coupled to the antinodes of the electromagnetic field confined in the cavity.

\section{ACKNOWLEDGMENTS}

The Authors acknowledge Andrea Fiore and Maurangelo Petruzzella (Eindhoven University of Technology) for their technical support to sample fabrication at the early stages of this work. M.F., G.P., F.B., and S.R. would like to acknowledge support and funding from the Italian Ministry for Education, University and Research within the Futuro in Ricerca (FIRB) program (project DeLIGHTeD, Protocol No. RBFR12RS1W). A.P. and M.F. also acknowledge funding from the Regione Lazio programme "Progetti di Gruppi di ricerca" legge Regionale No. 13/2008 (project SINFONIA, Protocol No. 85-2017-15200) via LazioInnova spa. This work also received support from Sapienza University of Rome, through Fondi Ateneo 2015-2019, as well as from the European Unions Horizon 2020 Research and Innovation Program under the Marie Sklodowska-Curie Innovative Training Network PROMIS (Postgraduate Research on Dilute Metamorphic Nanostructures and Metamaterials in Semiconductor Photonics), Grant Agreement No. 641899, Funder Id: http: //dx.doi.org/10.13039/100010665.

\section{APPENDIX A: EXCITON DYNAMICS IN A QD-PhC CAVITY SYSTEM. DETAILS ON THEORETICAL MODEL}

\section{Reservoir of electron-hole pairs}

As noted in the main text, the inclusion of a reservoir of electron-hole pairs-with population $f_{e, h}=e^{-\gamma_{\mathrm{c}} \cdot(t+\Delta \tau)}$, as defined in Eq. (3) - as the sole source of carriers for the QD is a crucial aspect of our model. In particular, the introduction of an offset between the time at which we set the initial conditions for our rate-equation system $(t=0)$ and the time at which the excitation occurs $(t=-\Delta \tau$; it should also be noted that $f_{e, h}$ is normalized to be equal to unity at the time of the excitation) is instrumental to our ability to accurately model photon correlation data. It must be noted, however, that the simplifications underlying the formulation of Eq. (3) are rather significant, as the correct time dependence of $f_{e, h}$ should result, in principle, from solving the appropriate system of rate equations, taking into account all the main processes potentially resulting in a variation of the reservoir's population. In strict terms, both radiative and nonradiative recombinations from the reservoir should be included in the model, as well as carrier transfer to and from all available states, obviously including - but not limited to - those of the QD. As a result, the temporal evolution of $f_{e, h}$ will likely differ, perhaps significantly, from the simple exponential function postulated in Eq. (3). However, as also noted in the main text, the assumptions made to define Eq. (3) can be effectively accounted for by employing different effective values of $\gamma_{c}$ the QD carrier capture rate-when using the solutions of Eq. (4) to fit different experiments. As already discussed in Sec. IIC, this is particularly true for time-resolved microPL and photon correlation measurements, which effectively probe the QD carrier feeding process over different time ranges.

\section{Inclusion of PhC cavity state}

According to the dissipative JC model $[48,70,76,77]$, the time evolution of the decisive entries of the density matrix of a 
two-level system coupled to a cavity mode (CM) are described by the following equations:

$$
\begin{aligned}
& \frac{d \rho_{a a}}{d t}=\frac{d \rho_{\mathrm{QD}}}{d t}=-g\left(\rho_{a b}+\rho_{a b}^{*}\right)-\gamma \rho_{\mathrm{QD}}, \\
& \frac{d \rho_{b b}}{d t}=\frac{d \rho_{\mathrm{CM}}}{d t}=g\left(\rho_{a b}+\rho_{a b}^{*}\right)-\kappa \rho_{\mathrm{CM}}, \\
& \frac{d \rho_{a b}}{d t}=g\left(\rho_{\mathrm{QD}}-\rho_{\mathrm{CM}}\right)-\left[\gamma_{\mathrm{tot}}+i(\Delta \mathrm{E} / \hbar)\right] \rho_{a b} .
\end{aligned}
$$

Given that the QD-if schematized as a two-level system - can either be empty (in the ground state, $g$ ) or filled with an exciton (in its excited state, $e$ ), while the cavity mode can be in the vacuum state $\left(0_{c}\right)$ or in a single-photon state $\left(1_{c}\right)$, the relevant states for the QD-cavity system are $|a\rangle=\left|e, 0_{c}\right\rangle$ and $|b\rangle=\left|g, 1_{c}\right\rangle$. Within this context, the diagonal elements of the density matrix, $\rho_{a a}$ and $\rho_{b b}$, represent the populations of the QD and of the CM, respectively (and are accordingly labeled as $\rho_{\mathrm{QD}}$ and $\rho_{\mathrm{CM}}$ ). If $\Gamma \ll \frac{\gamma_{\text {tot }}}{2}=\frac{\kappa+\gamma+2 \gamma_{\mathrm{dp}}}{4}$ there is no time to establish Rabi oscillations between the QD and the $\mathrm{CM}$; i.e., we are in the weak-coupling regime (as defined, for example, in Ref. [48]). Our system clearly respects this condition; in particular, $\kappa\left(760 \mathrm{~ns}^{-1}\right)$ is much larger than $\gamma$ $\left(\sim 1 \mathrm{~ns}^{-1}\right), \gamma_{\mathrm{dp}}\left(=6 \mathrm{~ns}^{-1}\right)$, and $\Gamma\left(\leqslant 15 \mathrm{~ns}^{-1}\right)$, so that we can write $\Gamma \ll \frac{\gamma_{\text {tot }}}{2} \sim \frac{\kappa}{4}$. As a result, the nondiagonal element of the density matrix, $\rho_{a b}$, varies very slowly with respect to $\rho_{\mathrm{QD}}$ and $\rho_{\mathrm{CM}}$, and we can adiabatically set

$$
\frac{d \rho_{a b}}{d t}=g\left(\rho_{\mathrm{QD}}-\rho_{\mathrm{CM}}\right)-\left[\gamma_{\mathrm{tot}}+i(\Delta \mathrm{E} / \hbar)\right] \rho_{a b}=0 .
$$

This equation yields

$$
\rho_{a b}=\frac{g}{\gamma_{\mathrm{tot}}+i(\Delta \mathrm{E} / \hbar)}\left(\rho_{\mathrm{QD}}-\rho_{\mathrm{CM}}\right),
$$

which results in the possibility to rewrite the equations describing the time evolution of $\rho_{\mathrm{QD}}$ and $\rho_{\mathrm{CM}}$ [see Eq. (A1)] as

$$
\begin{aligned}
\frac{d \rho_{\mathrm{QD}}}{d t} & =2 g^{2} \frac{\gamma_{\mathrm{tot}}}{\gamma_{\mathrm{tot}}^{2}+(\Delta \mathrm{E} / \hbar)^{2}}\left(\rho_{\mathrm{CM}}-\rho_{\mathrm{QD}}\right)-\gamma \rho_{\mathrm{QD}} \\
& =\Gamma \rho_{\mathrm{CM}}-(\gamma+\Gamma) \rho_{\mathrm{QD}}, \\
\frac{d \rho_{\mathrm{CM}}}{d t} & =2 g^{2} \frac{\gamma_{\mathrm{tot}}}{\gamma_{\mathrm{tot}}^{2}+(\Delta \mathrm{E} / \hbar)^{2}}\left(\rho_{\mathrm{QD}}-\rho_{\mathrm{CM}}\right)-\kappa \rho_{\mathrm{CM}} \\
& =\Gamma \rho_{\mathrm{QD}}-(\kappa+\Gamma) \rho_{\mathrm{CM}}
\end{aligned}
$$

[where we also used the definition of $\Gamma$ provided in Eq. (1)]. We have thus obtained the rate equations for a (two-level) QD weakly coupled to a CM, wherein if the QD exciton "transfers" to the CM, which happens with rate $\Gamma$, a photon is immediately (i.e., with rate $\kappa$, much larger than both $\gamma$ and $\Gamma$ ) emitted into the environment. In this regime, photons captured in the cavity do not live long enough to be reabsorbed by the QD, so that the transfer to the CM effectively serves as an additional radiative recombination channel for the exciton [70], with a Purcell-enhanced recombination rate that is approximately equal to $\Gamma_{\text {tot }}$ [as anticipated in Eq. (1)].

As noted above, Eq. (A4) is only valid if the QD can be described, at least approximately, as a two-level system. For a QD characterized by the states' ladder shown in Fig. 4(a), however, many of the assumptions underlying Eq. (A4) retain their validity, as long as a single -QD state is (weakly) coupled to the $\mathrm{CM}$. This is clearly the case for our QD-PhC cavity system, wherein only the single-exciton (X) state displays a significant coupling with the CM [as sketched in step 3 of Fig. 4(a)]. As discussed in the main text, under such conditions the time evolution of the system is well described by the solution of the system of rate equations provided in Eq. (4).

\section{APPENDIX B: PHOTON CORRELATION}

As introduced in Ref. [82], for positive time delays $(\tau \geqslant 0)$ and for continuous-wave $(\mathrm{CW})$ excitation $g_{i j-k l}^{(2)}(\tau)$ is nothing but the time dependence of the probability of observing the $k l$ transition, with $\tau=0$ corresponding to the moment at which the $i j$ transition is observed ( $i j$ and $k l$ are swapped for negative time delays). This of course implies that, for $\tau \geqslant 0(\tau \leqslant 0), g_{i j-k l}^{(2)}(\tau)$ is equal to $p_{k l}(\tau)\left[p_{i j}(-\tau)\right]$, provided that the initial conditions set for Eq. (4) are chosen to match the configuration of the system corresponding to the final state of the $i j(\mathrm{kl})$ transition. Following the recombination of the biexciton, for example, the QD is populated with an exciton, so that for $\tau \geqslant 0, g_{22-11}^{(2)}(\tau)$ [i.e., $g_{2 \mathrm{X}-\mathrm{X}}^{(2)}(\tau)$ ] is equal to $p_{11}(\tau)$, obtained solving Eq. (4) with initial conditions $p_{11}(\tau=0)=1$ [for $\tau \leqslant 0$ the relevant probability is of course $p_{22}(-\tau)$, and the system is initially in its empty state, i.e., $\left.p_{00}(\tau=0)=1\right]$.

In general, these considerations remain true if we focus on a $g_{i j-k l}^{(2)}(\tau)$ function obtained under pulsed, rather than $\mathrm{CW}$, excitation conditions. However, in this case, we must carefully consider every aspect of the interconnected chain of events progressively leading to the buildup of the $g_{i j-k l}^{(2)}(\tau)$ histogram. First of all, after excitation with a (deltalike) laser pulse, a photon corresponding to the $i j$ transition (again, if we focus on the $\tau \geqslant 0$ case) is emitted with a given time delay, $\Delta \tau$. Of course, the probability distribution of the value of $\Delta \tau$ is nothing but $p_{i j}(\Delta \tau)$, as obtained by solving Eq. (4) with $p_{00}(\Delta \tau=0)=1$. Afterwards a second photon, corresponding to the $k l$ transition, is emitted at a time $\tau$, with $\tau=0$ corresponding to the time at which the $i j$ photon is observed. As already noted, the probability that the $k l$ photon be emitted at $\tau, p_{k l}$, has to be computed by solving Eq. (4) for the initial conditions associated with the emission of an $i j$ photon at $\tau=0$. At this point, however, it is crucially important to note that the value of $\Delta \tau$-the delay between the laser pulse and the emission of the $i j$ photon, as discussed above-also influences the probability of emission of the $k l$ photon. As such, when computing $p_{k l}$ the correct value of $\Delta \tau$ must be included in the expression describing the population of carriers in the reservoir feeding our system, $f_{e, h}=$ $e^{-\gamma_{\mathrm{c}}(\tau+\Delta \tau)}$ [as defined by Eq. (3); see also Fig. 4(a) and related discussion]. Put in more formal terms, for each laser pulse $p_{k l}$ takes the form of a conditional probability, $p_{k l}(\tau \mid \Delta \tau)$; i.e., it is the probability that the $k l$ photon be emitted with a time delay $\tau$ with respect to the emission of the $i j$ photon, on the condition that the latter be emitted with a delay $\Delta \tau$ from the laser pulse. In turn, $p_{k l}(\tau \mid \Delta \tau)$ has to be averaged over a large number of laser pulses in order to correctly reconstruct a $g_{i j-k l}^{(2)}(\tau)$ function obtained under pulsed excitation; this implies averaging $p_{k l}(\tau \mid \Delta \tau)$ over all possible values of 


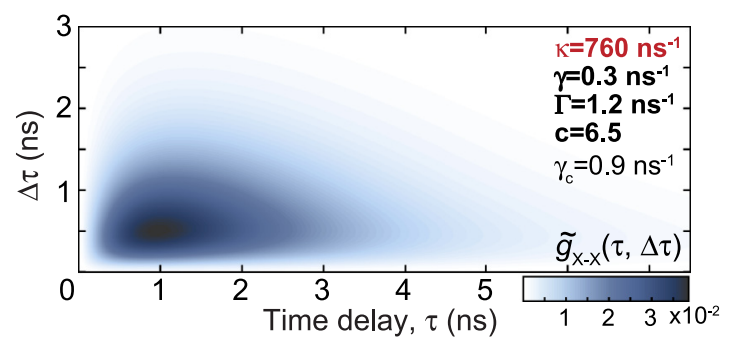

FIG. 8. Dependence on $\tau$ and $\Delta \tau$ of $\tilde{g}_{\mathrm{X}-\mathrm{X}}(\tau, \Delta \tau)$, as defined in Eq. (B1). In order to compute $\tilde{g}_{\mathrm{X}-\mathrm{X}}(\tau, \Delta \tau)$ we used the parameters extracted from fitting the data displayed in Fig. 5. The parameters' values are reported following the convention established in Fig. 4(b): $\kappa$, which is fixed throughout the fitting procedure, is reported in bold red; $\gamma, \Gamma$, and $c$, which are estimated from the time-resolved data (see Fig. 4) and then fixed when fitting photon-correlation measurements, are in bold; and $\gamma_{\mathrm{c}}$ is in plain text.

$\Delta \tau$, weighted with the appropriate probability distribution. This distribution is, of course, $p_{i j}(\Delta \tau)$ (see above), so that we have

$$
\begin{aligned}
g_{i j-k l}^{(2) \tau_{p}=0}(\tau) & =\int_{0}^{\infty} p_{k l}(\tau \mid \Delta \tau) p_{i j}(\Delta \tau) d \Delta \tau \\
& =\int_{0}^{\infty} \tilde{g}_{i j-k l}(\tau, \Delta \tau) d \Delta \tau .
\end{aligned}
$$

Figure 8 displays the dependence on $\tau$ and $\Delta \tau$ (for $\tau \geqslant$ 0 ) of $\tilde{g}_{i j-k l}(\tau, \Delta \tau)$, the integrand on the right-hand side of Eq. (B1). The example shown in the figure refers to the $i j=k l=11$ case, corresponding to the $\mathrm{X}-\mathrm{X}$ autocorrelation; the parameters chosen to compute $\tilde{g}_{\mathrm{X}-\mathrm{X}}(\tau, \Delta \tau)$ are those eXtracted from fitting the measured $g_{\mathrm{X}-\mathrm{X}}^{(2)}(\tau)$ function (see Fig. 5) to the model, as described below.

It is extremely important to note the presence of the " $\tau_{p}=$ 0 " label in Eq. (B1), to identify the correlation peak positioned at null time delays. Indeed, this expression only accounts for those laser pulses that result in the emission of two photons, thereby contributing to the presence of nonzero correlation values in the region centered around $\tau_{p}=0$. For larger time delays, $g_{i j-k l}^{(2)}(\tau)$ is characterized by the presence of a series of periodic peaks, centered at $\tau_{p}=n \tau_{\mathrm{Ti}: \mathrm{Sa}}(n=1,2, \cdots)$, where
$\tau_{\mathrm{Ti}: \mathrm{Sa}}=12.5 \mathrm{~ns}$ is the laser repetition period (see, for example, Fig. 5). These peaks, which are due to the observation of $i j-k l$ photon pairs generated by different laser pulses (of course spaced by $n \tau_{\mathrm{Ti}: \mathrm{Sa}}$ ), are well described by the expression

$$
g_{i j-k l}^{(2) \tau_{p}=n \tau_{\mathrm{T}: \mathrm{Sa}}}(\tau)=\int_{0}^{\infty} p_{k l}\left(\tau-n \tau_{\mathrm{Ti}: \mathrm{Sa}}+\tau^{\prime}\right) p_{i j}\left(-\tau^{\prime}\right) d \tau^{\prime},
$$

where $p_{k l}$ and $p_{i j}$ are both obtained by solving Eq. (4) with $p_{00}(0)=1$ and $\Delta \tau=0$. By combining Eqs. (B1) and (B2) we can finally obtain a general formulation of $g_{i j-k l}^{(2)}(\tau)$, valid for pulsed excitation and for an arbitrary pair of $i j-k l$ transitions:

$$
\begin{aligned}
& g_{i j-k l}^{(2)}(\tau)=g_{i j-k l}^{(2) \tau_{p}=0}(\tau)+\sum_{n=1}^{\infty} g_{i j-k l}^{(2) \tau_{p}=n \cdot \tau_{\mathrm{T}: \mathrm{Sa}}}(\tau) \text { for } \tau \geqslant 0, \\
& g_{i j-k l}^{(2)}(\tau)=g_{k l-i j}^{(2)}(-\tau) \text { for } \quad \tau \leqslant 0 .
\end{aligned}
$$

After convolution with the instrumental response [displayed, e.g., in Fig. 4(b)], the function provided in Eq. (B3) is ideally suited for fitting photon correlation measurements. It should be noted, in particular, that the proposed fitting function can accurately reproduce the peculiar "dip" characterizing the central autocorrelation peak displayed in Fig. 5. This dip is, actually, a pretty common occurrence in autocorrelation histograms; see, for example, Ref. [106]. Indeed, the generic excitonic state, which we can label as " $i j$," is univocally associated with the presence of a certain number of electrons $(i)$ and holes $(j)$ in the QD. However, the observation of a radiative transition associated with the $i j$ state invariably involves the recombination of an $e-h$ pair, i.e., a change in the QD occupation to $i-1$ electrons and $j-1$ holes. Immediately after the emission of the first $i j$ photon, therefore, the QD simply cannot be in the $i j$ state anymore; in turn, this implies that the probability of emitting a second $i j$ photon at the same time as the first one (i.e., for a time delay $\tau \equiv 0$ ) is strictly zero. This will result in the presence of an observable dip in the experimental $g_{i j-i j}^{(2)}(\tau)$, as long as the QD recombination dynamics is slower than the detectors' resolution. This is the case for the QD-PhC cavity system displayed in Fig. 5, for which the presence of a fairly large QD-PhC cavity detuning $(4.1 \mathrm{meV})$ leads to a sizable slowdown of the exciton recombination dynamics.
[1] P. Michler, A. Kiraz, C. Becher, W. V. Schoenfeld, P. M. Petroff, L. D. Zhang, E. Hu, and A. Imamoğlu, Science 290, 2282 (2000).

[2] Y.-J. Wei, Y.-M. He, M.-C. Chen, Y.-N. Hu, Y. He, D. Wu, C. Schneider, M. Kamp, S. Höfling, C.-Y. Lu, and J.-W. Pan, Nano Lett. 14, 6515 (2014).

[3] N. Somaschi, V. Giesz, L. De Santis, J. C. Loredo, M. P. Almeida, G. Hornecker, S. L. Portalupi, T. Grange, C. Antón, J. Demory, C. Gómez, I. Sagnes, N. D. Lanzillotti-Kimura, A. L. Lemaítre, A. Auffèves, A. G. White, L. Lanco, and P. Senellart, Nat. Photonics 10, 340 (2016).
[4] C. L. Salter, R. M. Stevenson, I. Farrer, C. A. Nicoll, D. A. Ritchie, and A. J. Shields, Nature 465, 594 (2010).

[5] G. Juska, V. Dimastrodonato, L. O. Mereni, A. Gocalinska, and E. Pelucchi, Nat. Photonics 7, 527 (2013).

[6] M. Müller, S. Bounouar, K. D. Jöns, M. Glässl, and P. Michler, Nat. Photonics 8, 224 (2014).

[7] R. Trotta, J. S. Wildmann, E. Zallo, O. G. Schmidt, and A. Rastelli, Nano Lett. 14, 3439 (2014).

[8] J. Zhang, J. S. Wildmann, F. Ding, R. Trotta, Y. Huo, E. Zallo, D. Huber, A. Rastelli, and O. G. Schmidt, Nat. Commun. 6, 10067 (2015). 
[9] M. N. Makhonin, J. E. Dixon, R. J. Coles, B. Royall, I. J. Luxmoore, E. Clarke, M. Hugues, M. S. Skolnick, and A. M. Fox, Nano Lett. 14, 6997 (2014).

[10] C. Bentham, I. E. Itskevich, R. J. Coles, B. Royall, E. Clarke, J. O'Hara, N. Prtljaga, A. M. Fox, M. S. Skolnick, and L. R. Wilson, Appl. Phys. Lett. 106, 221101 (2015).

[11] C. P. Dietrich, A. Fiore, M. G. Thompson, M. Kamp, and S. Höfling, Laser Photonics Rev. 10, 870 (2016).

[12] A. Crespi, R. Osellame, R. Ramponi, D. J. Brod, E. F. Galvao, N. Spagnolo, C. Vitelli, E. Maiorino, P. Mataloni, and F. Sciarrino, Nat. Photonics 7, 545 (2013).

[13] K. Hennessy, A. Badolato, M. Winger, D. Gerace, M. Atatüre, S. Gulde, S. Fält, E. L. Hu, and A. Imamoğlu, Nature 445, 896 (2007).

[14] P. Gallo, M. Felici, B. Dwir, K. A. Atlasov, K. F. Karlsson, A. Rudra, A. Mohan, G. Biasiol, L. Sorba, and E. Kapon, Appl. Phys. Lett. 92, 263101 (2008).

[15] M. Nomura, N. Kumagai, S. Iwamoto, Y. Ota, and Y. Arakawa, Nat. Phys. 6, 279 (2010).

[16] D. Dalacu, K. Mnaymneh, V. Sazonova, P. J. Poole, G. C. Aers, J. Lapointe, R. Cheriton, A. J. SpringThorpe, and R. Williams, Phys. Rev. B 82, 033301 (2010).

[17] M. Calic, P. Gallo, M. Felici, K. A. Atlasov, B. Dwir, A. Rudra, G. Biasiol, L. Sorba, G. Tarel, V. Savona, and E. Kapon, Phys. Rev. Lett. 106, 227402 (2011).

[18] C. Jarlov, É. Wodey, A. Lyasota, M. Calic, P. Gallo, B. Dwir, A. Rudra, and E. Kapon, Phys. Rev. Lett. 117, 076801 (2016).

[19] E. T. Jaynes and F. W. Cummings, IEEE Proc. 51, 89 (1963).

[20] M. Felici, P. Gallo, A. Mohan, B. Dwir, A. Rudra, and E. Kapon, Small 5, 938 (2009).

[21] D. Dalacu, M. E. Reimer, S. Fréderick, D. Kim, J. Lapointe, P. J. Poole, G. C. Aers, R. L. Williams, W. Ross McKinnon, M. Korkusinski, and P. Hawrylak, Laser Photonics Rev. 4, 283 (2009).

[22] A. Mohan, P. Gallo, M. Felici, B. Dwir, A. Rudra, J. Faist, and E. Kapon, Small 6, 1268 (2010).

[23] A. Huggenberger, S. Heckelmann, C. Schneider, S. Höfling, S. Reitzenstein, L. Worschech, M. Kamp, and A. Forchel, Appl. Phys. Lett. 98, 131104 (2011).

[24] A. Schramm, J. Tommila, C. Strelow, T. V. Hakkarainen, A. Tukiainen, M. Dumitrescu, A. Mews, T. Kipp, and M. Guina, Nanotechnology 23, 175701 (2012).

[25] K. D. Jöns, P. Atkinson, M. Müller, M. Heldmaier, S. M. Ulrich, O. G. Schmidt, and P. Michler, Nano Lett. 13, 126 (2013).

[26] B. Liang, P.-S. Wong, T. Tran, V. G. Dorogan, Y. I. Mazur, M. E. Ware, G. J. Salamo, C.-K. Shih, and D. L. Huffaker, Nano Res. 6, 235 (2013).

[27] M. J. Holmes, K. Choi, S. Kako, M. Arita, and Y. Arakawa, Nano Lett. 14, 982 (2014).

[28] A. Surrente, R. Carron, P. Gallo, A. Rudra, B. Dwir, and E. Kapon, Nano Res. 9, 3279 (2016).

[29] A. Surrente, M. Felici, P. Gallo, A. Rudra, B. Dwir, and E. Kapon, Appl. Phys. Lett. 111, 221102 (2017).

[30] M. Felici, A. Polimeni, G. Salviati, L. Lazzarini, N. Armani, F. Masia, M. Capizzi, F. Martelli, M. Lazzarino, G. Bais, M. Piccin, S. Rubini, and A. Franciosi, Adv. Mater. 18, 1993 (2006).
[31] R. Trotta, A. Polimeni, F. Martelli, G. Pettinari, M. Capizzi, L. Felisari, S. Rubini, M. Francardi, A. Gerardino, P. C. M. Christianen, and J. C. Maan, Adv. Mater. 23, 2706 (2011).

[32] R. Trotta, A. Polimeni, and M. Capizzi, Adv. Funct. Mater. 22, 1782 (2012).

[33] M. Felici, S. Birindelli, R. Trotta, M. Francardi, A. Gerardino, A. Notargiacomo, S. Rubini, F. Martelli, M. Capizzi, and A. Polimeni, Phys. Rev. Appl. 2, 064007 (2014).

[34] M. Du, S. Limpijumnong, and S. Zhang, Phys. Rev. B 72, 073202 (2005).

[35] L. Wen, M. Stavola, W. B. Fowler, R. Trotta, A. Polimeni, M. Capizzi, G. Bisognin, M. Berti, S. Rubini, and F. Martelli, Phys. Rev. B 86, 085206 (2012).

[36] G. Baldassarri, M. Bissiri, A. Polimeni, M. Capizzi, M. Fischer, M. Reinhardt, and A. Forchel, Appl. Phys. Lett. 78, 3472 (2001).

[37] P. J. Klar, H. Grüning, M. Güngerich, W. Heimbrodt, J. Koch, T. Torunski, W. Stolz, A. Polimeni, and M. Capizzi, Phys. Rev. B 67, 121206(R) (2003).

[38] I. Buyanova, M. Izadifard, I. Ivanov, J. Birch, W. Chen, M. Felici, A. Polimeni, M. Capizzi, Y. Hong, H. Xin, and C. Tu, Phys. Rev. B 70, 245215 (2004).

[39] G. Bisognin, D. De Salvador, A. V. Drigo, E. Napolitani, A. Sambo, M. Berti, A. Polimeni, M. Felici, M. Capizzi, M. Guengerich, and P. J. Klar, Appl. Phys. Lett. 89, 061904 (2006).

[40] M. Berti, G. Bisognin, D. De Salvador, E. Napolitani, S. Vangelista, A. Polimeni, M. Capizzi, F. Boscherini, G. Ciatto, S. Rubini, F. Martelli, and A. Franciosi, Phys. Rev. B 76, 205323 (2007).

[41] G. Ciatto, Hydrogenated Dilute Nitride Semiconductors (Pan Stanford Publishing, Singapore, 2015).

[42] S. Birindelli, M. Felici, J. S. Wildmann, A. Polimeni, M. Capizzi, A. Gerardino, S. Rubini, F. Martelli, A. Rastelli, and R. Trotta, Nano Lett. 14, 1275 (2014).

[43] F. Biccari, A. Boschetti, G. Pettinari, F. La China, M. Gurioli, F. Intonti, A. Vinattieri, M. S. Sharma, M. Capizzi, A. Gerardino, L. Businaro, M. Hopkinson, A. Polimeni, and M. Felici, Adv. Mater. 30, 1705450 (2018).

[44] G. Pettinari, L. A. Labbate, M. S. Sharma, S. Rubini, A. Polimeni, and M. Felici, Nanophotonics 8, 1465 (2019).

[45] G. Tarel and V. Savona, Phys. Rev. B 81, 075305 (2010).

[46] P. Kaer, T. Nielsen, P. Lodahl, A. P. Jauho, and J. Mørk, Phys. Rev. B 86, 085302 (2012).

[47] P. Kaer, T. R. Nielsen, P. Lodahl, A. P. Jauho, and J. Mørk, Phys. Rev. Lett. 104, 157401 (2010).

[48] K. H. Madsen, S. Ates, T. Lund-Hansen, A. Loeffler, S. Reitzenstein, A. Forchel, and P. Lodahl, Phys. Rev. Lett. 106, 233601 (2011).

[49] K. H. Madsen, P. Kaer, A. Kreiner-Moller, S. Stobbe, A. Nysteen, J. Mørk, and P. Lodahl, Phys. Rev. B 88, 045316 (2013).

[50] G. Pettinari, A. Gerardino, L. Businaro, A. Polimeni, M. Capizzi, M. Hopkinson, S. Rubini, F. Biccari, F. Intonti, A. Vinattieri, M. Gurioli, and M. Felici, Microelectron. Eng. 174, 16 (2017).

[51] R. Trotta, D. Giubertoni, A. Polimeni, M. Bersani, M. Capizzi, F. Martelli, S. Rubini, G. Bisognin, and M. Berti, Phys. Rev. B 80, 195206 (2009). 
[52] Y. Akahane, T. Asano, B. Song, and S. Noda, Appl. Phys. Lett. 83, 1512 (2003).

[53] K. A. Atlasov, M. Felici, K. F. Karlsson, P. Gallo, A. Rudra, B. Dwir, and E. Kapon, Opt. Express 18, 117 (2010).

[54] M. Felici, K. A. Atlasov, A. Surrente, and E. Kapon, Phys. Rev. B 82, 115118 (2010).

[55] L. C. Andreani and D. Gerace, Phys. Rev. B 73, 235114 (2006).

[56] P. J. Poole, K. Kaminska, P. Barrios, Z. Lu, and J. Liu, J. Cryst. Growth. 311, 1482 (2009).

[57] L. Seravalli, G. Trevisi, P. Frigeri, F. Rossi, E. Buffagni, and C. Ferrari, J. Phys. D: Appl. Phys. 46, 315101 (2013).

[58] E. Pelucchi, S. Watanabe, K. Leifer, Q. Zhu, B. Dwir, P. De Los Rios, and E. Kapon, Nano Lett. 7, 1282 (2007).

[59] D. G. Gevaux, A. J. Bennett, R. M. Stevenson, A. J. Shields, P. Atkinson, J. Griffiths, D. Anderson, G. A. C. Jones, and D. A. Ritchie, Appl. Phys. Lett. 88, 131101 (2006).

[60] A. Polimeni, M. Bissiri, A. Augieri, G. Baldassarri Hoger von Högersthal, M. Capizzi, D. Gollub, M. Fischer, M. Reinhardt, and A. Forchel, Phys. Rev. B 65, 235325 (2002).

[61] D. Valente, J. Suffczyński, T. Jakubczyk, A. Dousse, A. Lemaitre, I. Sagnes, L. Lanco, P. Voisin, A. Auffèves, and P. Senellart, Phys. Rev. B 89, 041302 (2014).

[62] J. I. Pankove, Optical Processes in Semiconductors (Dover, New York, 1975).

[63] E. M. Purcell, Phys. Rev. 69, 681 (1946).

[64] A. Högele, S. Seidl, M. Kroner, K. Karrai, R. J. Warburton, B. D. Gerardot, and P. M. Petroff, Phys. Rev. Lett. 93, 217401 (2004).

[65] A. Berthelot, I. Favero, G. Cassabois, C. Voisin, C. Delalande, P. Roussignol, R. Ferreira, and J.-M. Gérard, Nat. Phys. 2, 759 (2006).

[66] I. Favero, A. Berthelot, G. Cassabois, C. Voisin, C. Delalande, P. Roussignol, R. Ferreira, and J. Gérard, Phys. Rev. B 75, 073308 (2007).

[67] J. Houel, A. V. Kuhlmann, L. Greuter, F. Xue, M. Poggio, R. J. Warburton, B. D. Gerardot, P. A. Dalgarno, A. Badolato, P. M. Petroff, A. Ludwig, D. Reuter, and A. D. Wieck, Phys. Rev. Lett. 108, 107401 (2012).

[68] K. Matsuda, K. Ikeda, T. Saiki, H. Tsuchiya, H. Saito, and K. Nishi, Phys. Rev. B 63, 121304 (2001).

[69] L. Besombes, K. Kheng, L. Marsal, and H. Mariette, Phys. Rev. B 63, 155307 (2001).

[70] K. H. Madsen and P. Lodahl, New J. Phys. 15, 025013 (2013).

[71] L. O. Mereni, V. Dimastrodonato, R. J. Young, and E. Pelucchi, Appl. Phys. Lett. 94, 223121 (2009).

[72] T. Braun, C. Schneider, S. Maier, R. Igusa, S. Iwamoto, A. Forchel, S. Höfling, Y. Arakawa, and M. Kamp, AIP Adv. 4, 097128 (2014).

[73] A. Thoma, P. Schnauber, M. Gschrey, M. Seifried, J. Wolters, J. H. Schulze, A. Strittmatter, S. Rodt, A. Carmele, A. Knorr, T. Heindel, and S. Reitzenstein, Phys. Rev. Lett. 116, 033601 (2016).

[74] N. Ha, T. Mano, Y.-L. Chou, Y.-N. Wu, S.-J. Cheng, J. Bocquel, P. M. Koenraad, A. Ohtake, Y. Sakuma, K. Sakoda, and T. Kuroda, Phys. Rev. B 92, 075306 (2015).

[75] D. Huber, M. Reindl, Y. Huo, H. Huang, J. S. Wildmann, O. G. Schmidt, A. Rastelli, and R. Trotta, Nat. Commun. 8, 15506 (2017).
[76] P. Lodahl, S. Mahmoodian, and S. Stobbe, Rev. Mod. Phys. 87, 347 (2015).

[77] H. J. Carmichael, R. J. Brecha, M. G. Raizen, H. J. Kimble, and P. R. Rice, Phys. Rev. A 40, 5516 (1989).

[78] M. Winger, T. Volz, G. Tarel, S. Portolan, A. Badolato, K. J. Hennessy, E. L. Hu, A. Beveratos, J. Finley, V. Savona, and A. Imamoğlu, Phys. Rev. Lett. 103, 207403 (2009).

[79] N. Chauvin, C. Zinoni, M. Francardi, A. Gerardino, L. Balet, B. Alloing, L. H. Li, and A. Fiore, Phys. Rev. B 80, 241306 (2009).

[80] A. Auffèves, D. Gerace, J.-M. Gérard, M. F. Santos, L. C. Andreani, and J.-P. Poizat, Phys. Rev. B 81, 245419 (2010).

[81] R. Hanbury Brown and R. Q. Twiss, Nature 177, 27 (1956).

[82] M. H. Baier, A. Malko, E. Pelucchi, D. Y. Oberli, and E. Kapon, Phys. Rev. B 73, 205321 (2006).

[83] D. F. Walls and G. J. Milburn, Quantum Optics (SpringerVerlag, Berlin, Heidelberg, 2008).

[84] C. Jarlov, A. Lyasota, L. Ferrier, P. Gallo, B. Dwir, A. Rudra, and E. Kapon, Appl. Phys. Lett. 107, 191101 (2015).

[85] F. Adler, M. Geiger, A. Bauknecht, F. Scholz, H. Schweizer, M. H. Pilkuhn, B. Ohnesorge, and A. Forchel, J. Appl. Phys. 80, 4019 (1996).

[86] D. Birkedal, K. Leosson, and J. M. Hvam, Superlattices Microstruct. 31, 97 (2002).

[87] V. B. Verma, M. J. Stevens, K. L. Silverman, N. L. Dias, A. Garg, J. J. Coleman, and R. P. Mirin, Opt. Express 19, 4182 (2011).

[88] B. Patton, W. Langbein, and U. Woggon, Phys. Rev. B 68, 125316 (2003).

[89] C. Zinoni, B. Alloing, C. Monat, V. Zwiller, L. H. Li, A. Fiore, L. Lunghi, A. Gerardino, H. de Riedmatten, H. Zbinden, and N. Gisin, Appl. Phys. Lett. 88, 131102 (2006).

[90] A. R. A. Chalcraft, S. Lam, D. O'Brien, T. F. Krauss, M. Sahin, D. Szymanski, D. Sanvitto, R. Oulton, M. S. Skolnick, A. M. Fox, D. M. Whittaker, H. Y. Liu, and M. Hopkinson, Appl. Phys. Lett. 90, 241117 (2007).

[91] S. Strauf, K. Hennessy, M. Rakher, Y. Choi, A. Badolato, L. Andreani, E. Hu, P. Petroff, and D. Bouwmeester, Phys. Rev. Lett. 96, 127404 (2006).

[92] T. Yoshle, A. Scherer, J. Hendrickson, G. Khitrova, H. M. Gibbs, G. Rupper, C. Ell, O. B. Shchekin, and D. G. Deppe, Nature 432, 200 (2004).

[93] G. Khitrova, H. Gibbs, M. Kira, S. Koch, and A. Scherer, Nat. Phys. 2, 81 (2006).

[94] I. J. Luxmoore, N. A. Wasley, A. J. Ramsay, A. C. T. Thijssen, R. Oulton, M. Hugues, S. Kasture, V. G. Achanta, A. M. Fox, and M. S. Skolnick, Phys. Rev. Lett. 110, 037402 (2013).

[95] M. Schwartz, U. Rengstl, T. Herzog, M. Paul, J. Kettler, S. L. Portalupi, M. Jetter, and P. Michler, Opt. Express 24, 3089 (2016).

[96] J. P. Sprengers, A. Gaggero, D. Sahin, S. Jahanmirinejad, G. Frucci, F. Mattioli, R. Leoni, J. Beetz, M. Lermer, M. Kamp, S. Höfling, R. Sanjines, and A. Fiore, Appl. Phys. Lett. 99, 181110 (2011).

[97] S. L. Portalupi, G. Hornecker, V. R. Giesz, T. Grange, A. Lema tre, J. Demory, I. Sagnes, N. D. Lanzillotti-Kimura, L. C. Lanco, A. Auff ves, and P. Senellart, Nano Lett. 15, 6290 (2015).

[98] J. Iles-Smith, D. P. S. McCutcheon, A. Nazir, and J. Mork, Nat. Photonics 11, 521 (2017). 
[99] T. Grange, N. Somaschi, C. Antón, L. De Santis, G. Coppola, V. Giesz, A. Lemaître, I. Sagnes, A. Auffèves, and P. Senellart, Phys. Rev. Lett. 118, 253602 (2017).

[100] J. Liu, R. Su, Y. Wei, B. Yao, S. F. C. da Silva, Y. Yu, J. Iles-Smith, K. Srinivasan, A. Rastelli, J. Li, and X. Wang, Nat. Nanotechnol. 14, 586 (2019).

[101] A. J. Hudson, R. M. Stevenson, A. J. Bennett, R. J. Young, C. A. Nicoll, P. Atkinson, K. Cooper, D. A. Ritchie, and A. J. Shields, Phys. Rev. Lett. 99, 266802 (2007).
[102] M. Reindl, K. D. Jöns, D. Huber, C. Schimpf, Y. Huo, V. Zwiller, A. Rastelli, and R. Trotta, Nano Lett. 17, 4090 (2017). [103] M. Minkov and V. Savona, Phys. Rev. B 87, 125306 (2013).

[104] A. D. Greentree, C. Tahan, J. H. Cole, and L. C. L. Hollenberg, Nat. Phys. 2, 856 (2006).

[105] W. Xue, Y. Yu, L. Ottaviano, Y. Chen, E. Semenova, K. Yvind, and J. Mork, Phys. Rev. Lett. 116, 063901 (2016).

[106] T. Aichele, V. Zwiller, and O. Benson, New J. Phys. 6, 90 (2004). 